\title{
On the structure of conjugation-free fundamental groups of conic-line arrangements
}

\author{
Michael Friedman • David Garber
}

Received: 28 April 2013 / Accepted: 20 April 2014 / Published online: 27 August 2014

(C) Tbilisi Centre for Mathematical Sciences 2014

\begin{abstract}
The fundamental group of the complement of a hyperplane arrangement plays an important role in studying the corresponding arrangements. In particular, for large families of hyperplane arrangements, this fundamental group, being isomorphic to the fundamental group of a complement of a line arrangement, has some remarkable properties: either it is a direct sum of free groups and a free abelian group, or it has a conjugation-free geometric presentation. In this paper, we first give a complete proof to the following key lemma: if we draw a new line through only one intersection point of a given real line arrangement whose fundamental group is conjugation-free, then the fundamental group of the new arrangement is also conjugation-free. Second, we generalize this lemma to the case of conic-line arrangements. Moreover, we prove that once the graph associated to conic-line arrangements (defined slightly different than the corresponding graph for line arrangements) has no cycles, then the fundamental group of its complement has a conjugation-free geometric presentation and in addition can be written as a direct sum of free groups and a free abelian group. Also, we show that if the graph consists of one cycle, and the conic does not pass through all the multiple points corresponding to the vertices of the cycle, then the fundamental group has a conjugation-free geometric presentation as well. For conclusion, we extend the family of real line arrangements having a conjugation-free geometric presentation (for
\end{abstract}

Communicated by Shmuel Weinberger.

M. Friedman

Image Knowledge Gestaltung. An Interdisciplinary Laboratory, Cluster of Excellence,

Humboldt-Universität zu Berlin, Unter den Linden 6, 10099 Berlin, Germany

e-mail: michael.friedman@hu-berlin.de

D. Garber $(\bowtie)$

Department of Applied Mathematics, Faculty of Sciences, Holon Institute of Technology,

52 Golomb St., PO Box 305, 5810201 Holon, Israel

e-mail: garber@hit.ac.il 
their fundamental group) by defining the notion of a conjugation-free graph. We also extend this notion to certain families of conic-line arrangements.

Keywords Fundamental group · Hyperplane arrangement · Conic-line arrangement · Conjugation-free presentation · Conjugation-free graph

\section{Introduction}

The fundamental group of the complement of a plane curve is a very important topological invariant. For example, it is used to distinguish between curves that form a Zariski pair, which is a pair of curves having the same combinatorics but non-homeomorphic complements in $\mathbb{C P}^{2}$ (see [6] for the exact definition and [7] for a survey). Another example is that while the fundamental group of the complement of a nodal curve is abelian (see [31]), there are curves with non-abelian fundamental groups. Thus, it is interesting to explore finite non-abelian groups which arise that way, see for example $[4,5,8,30]$.

Moreover, the Zariski-Lefschetz hyperplane section theorem (see [21]) states that $\pi_{1}\left(\mathbb{C P}^{N}-S\right) \cong \pi_{1}(H-(H \cap S))$, where $S$ is a hypersurface and $H$ is a generic 2-plane. Since $H \cap S$ is a plane curve, the fundamental groups of complements of plane curves can also be used for computing the fundamental groups of complements of hypersurfaces in $\mathbb{C P}^{N}$. Note that when $S$ is a hyperplane arrangement, $H \cap S$ is a line arrangement in $\mathbb{C P}^{2}$. Thus, one of the main tools for investigating the topology of hyperplane arrangements is the fundamental groups $\pi_{1}\left(\mathbb{C P}^{2}-\mathcal{L}\right)$ and $\pi_{1}\left(\mathbb{C}^{2}-\mathcal{L}\right)$, where $\mathcal{L}$ is a line arrangement.

For line arrangements these groups have very interesting properties (see e.g. [26, Section 5.3]). They are abelian if and only if $\mathcal{L}$ has only nodes as intersection points, see for example [9, Example 1.6(a)]. Moreover, Fan [14] and Eliyahu et al. [12] proved that this group is a direct sum of a free abelian group and free groups if and only if a certain graph, associated to the intersection points of $\mathcal{L}$ (see Sect. 2), has no cycles. Based on this, Eliyahu et al. $[10,11]$ showed that other properties hold for certain presentations of this group: conjugation-free and complemented presentations.

As conic-line arrangements are a natural generalization of line arrangements, an immediate question that arises is whether the above properties (e.g. conjugationfreeness or a structure of a direct sum of a free abelian group and free groups) hold for these arrangements too. One should note, contrary to the situation for line arrangements, that this group can be abelian even if the conic-line arrangement has singular points which are not nodes (see [8] and Fig. 3 below).

Note that the fundamental groups $\pi_{1}\left(\mathbb{C P}^{2}-\mathcal{A}\right)$ and $\pi_{1}\left(\mathbb{C}^{2}-\mathcal{A}\right)$, for some families of conic-line arrangements $\mathcal{A}$, were studied by Amram et al. (see e.g. [1,2] and especially [3, Theorem 6]). Moreover, Zariski pairs consisting of conic-line arrangements were studied by, for example, Namba-Tsuchihashi [24] and Tokunaga [27], but a research in the spirit of the above questions has not been carried out yet.

In this paper, we generalize Fan's result to the case of conic-line arrangements. After surveying in Sect. 2 the known results on line arrangements, the braid mon- 
odromy technique and the conjugation-free property, Sect. 3 deals with the preservation of this property under certain actions for line arrangements, which corrects and completes the proofs given in [11]. Section 4 examines this property for conicline arrangements. In Sect. 5.1, we present a necessary condition which implies that the fundamental group of some families of conic-line arrangements is a direct sum of a free abelian group and free groups. Explicitly, we generalize Fan's concept of a graph associated to line arrangements to the case of real conic-line arrangements and prove that once this graph has no cycles, then the corresponding fundamental group has the desired structure (for an explicit formulation, see Theorem 2.7). In Sect. 5.2, we prove a few propositions about the structure of the fundamental group of a conic-line arrangement whose graph consists of a single cycle, where the conic does not pass through all the multiple points corresponding to the vertices of the cycle.

In Sect. 6, we define the notion of a conjugation-free graph, for both line arrangements and conic-line arrangements. We show that for every arrangement whose graph is a conjugation-free graph, the fundamental group of its complement has a conjugation-free geometric presentation.

\section{Arrangements, braid monodromy and conjugation-free property}

In this section, we give a short survey of some known results concerning the structure of the fundamental group of the complement of a line arrangement. After that, we present the family of conic-line arrangements that we deal with and give a short survey about the braid monodromy technique, for computing presentations of fundamental groups of complements of plane curves. In the last subsection, we present the notion of conjugation-free geometric presentation of a fundamental group associated to a line arrangement or to a conic-line arrangement.

\subsection{Line arrangements and conic-line arrangements}

An affine line arrangement in $\mathbb{C}^{2}$ is a union of copies of $\mathbb{C}^{1}$ in $\mathbb{C}^{2}$. Such an arrangement is called real if the defining equations of all its lines can be written with real coefficients, and complex otherwise.

For real and complex line arrangements $\mathcal{L}$, Fan [14] defined a graph $G(\mathcal{L})$ which is associated to its multiple points (i.e. points where more than two lines are intersected). We give here its version for real arrangements (the general version is more delicate to explain and will be omitted): Given a real line arrangement $\mathcal{L}$, the graph $G(\mathcal{L})$ of multiple points lies on the real part of $\mathcal{L}$. It consists of the multiple points of $\mathcal{L}$ as vertices, with the segments between the multiple points on lines which have at least two multiple points as edges. Note that if the arrangement consists of three multiple points on the same line, then $G(\mathcal{L})$ has three vertices on the same edge (see Fig. 1a). If two such lines happen to intersect in a simple point (i.e. a point where exactly two lines are intersected), it is ignored (i.e. there is no corresponding vertex in the graph). See another example in Fig. 1b (note that Fan's definition gives a graph slightly different from the graph defined in $[20,29])$. 


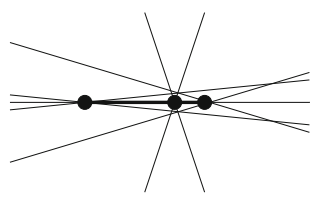

(a)

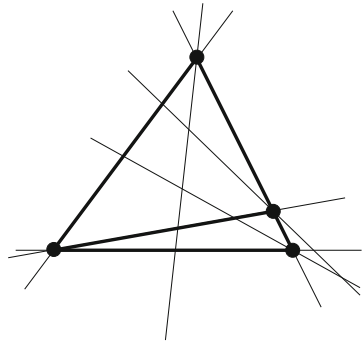

(b)

Fig. 1 Examples for the graph $G(\mathcal{L})$

Fan $[13,14]$ proved the following result:

Proposition 2.1 (Fan) Let $\mathcal{L}$ be a complex arrangement of $k$ lines and $S=\left\{a_{1}, \ldots, a_{p}\right\}$ be the set of all multiple points of $\mathcal{L}$. Suppose that $\beta(\mathcal{L})=0$, where $\beta(\mathcal{L})$ is the first Betti number of the graph $G(\mathcal{L})$ (hence $\beta(\mathcal{L})=0$ means that the graph $G(\mathcal{L})$ has no cycles). Then:

$$
\pi_{1}\left(\mathbb{C}^{2}-\mathcal{L}\right) \cong \mathbb{Z}^{r} \oplus \bigoplus_{i=1}^{p} \mathbb{F}_{m\left(a_{i}\right)-1}
$$

where $m\left(a_{i}\right)$ is the multiplicity of the intersection point $a_{i}$ and $r=k+p-\sum_{i=1}^{p} m\left(a_{i}\right)$.

Remark 2.2 In fact Fan proved the above proposition for the projective fundamental group; however, the derivation for the affine group is trivial.

Eliyahu et al. [12] proved the inverse direction to Fan's result (which was conjectured by Fan [14]), i.e. if the fundamental group of the arrangement is a direct sum of free groups and a free abelian group, then the associated graph has no cycles.

We will generalize Fan's result to real conic-line arrangements. We start by defining them.

Definition 2.3 A real conic-line (CL) arrangement $\mathcal{A}$ is a union of conics and lines in $\mathbb{C}^{2}$, where all the conics and the lines are defined over $\mathbb{R}$ and every singular point (with respect to a generic projection) of the arrangement is in $\mathbb{R}^{2}$. In addition, for every conic $C \in \mathcal{A}, C \cap \mathbb{R}^{2}$ is not an empty set, neither a point nor a (double) line.

Moreover, we assume from now on the following assumption:

Assumption 2.4 Let $\mathcal{A}$ be a real CL arrangement. Then, for each pair of components $h_{1}, h_{2}$ of $\mathcal{A}, h_{1}$ and $h_{2}$ intersect transversally (i.e. the intersection multiplicity of $h_{1}, h_{2}$ is 1 at each intersection point).

For example, a tangency point is not permitted.

Remark 2.5 (1) We assume that no line passes through the branch points of the conics with respect to a generic projection. 


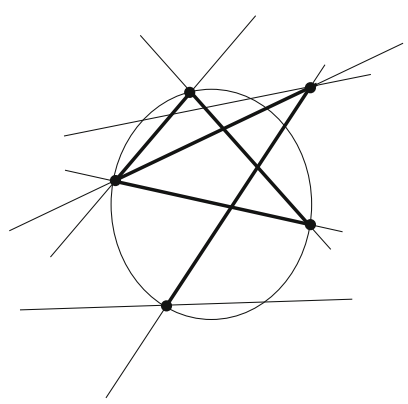

Fig. 2 An example for the graph $G(\mathcal{A})$ for a CL arrangement $\mathcal{A}$

(2) As every singular point of a real CL arrangement is in $\mathbb{R}^{2}$, the conics in the arrangements we deal with are either ellipses or hyperbolas, but not parabolas, since the second branch point of a parabola is at infinity.

Similar to Fan's graph for line arrangements, one can associate the following graph to a real CL arrangement:

Definition 2.6 The graph $G(\mathcal{A})$ for a real CL arrangement $\mathcal{A}$ is defined as follows: its vertices will be the multiple points (with multiplicity larger than 2), and its edges will be the segments on the lines connecting these points if two such points are on the same line (see an example in Fig. 2).

Then, one of the main results of this paper is:

Theorem 2.7 Let $\mathcal{A}$ be a real $C L$ arrangement with one conic and $k$ lines, and $S=\left\{a_{1}, \ldots, a_{p}, b_{1}, \ldots, b_{q}\right\}$ be the set of all multiple points of $\mathcal{A}$, where the conic is passing through the intersection points $a_{1}, \ldots, a_{p}$. Suppose that $\beta(\mathcal{A})=0$, where $\beta(\mathcal{A})$ is the first Betti number of the graph $G(\mathcal{A})$ (hence $\beta(\mathcal{A})=0$ means that the graph $G(\mathcal{A})$ has no cycles). Then:

$$
\pi_{1}\left(\mathbb{C}^{2}-\mathcal{A}\right) \cong \mathbb{Z}^{r} \oplus \bigoplus_{i=1}^{p} \mathbb{F}_{m\left(a_{i}\right)-2} \oplus \bigoplus_{i=1}^{q} \mathbb{F}_{m\left(b_{i}\right)-1}
$$

where $m(x)$ is the multiplicity of the singular point $x$ and $r=k+2 p+q+1-\sum_{i=1}^{p} m\left(a_{i}\right)-\sum_{i=1}^{q} m\left(b_{i}\right)$.

Note that while for line arrangements the inverse direction (i.e. such a structure of the fundamental group implies that the associated graph has no cycles) is correct [12], for CL arrangements it is not true anymore. For example, take three generic lines and a circle passing through the three intersection points (see Fig. 3a). Then, the fundamental group of the complement of this arrangement is abelian [8], although the first Betti number of the graph is 1 . We generalize this phenomenon in [15], showing that for a CL arrangement whose associated graph is a cycle of odd length and the conic passes through all the multiple points corresponding to the vertices of the cycle and 


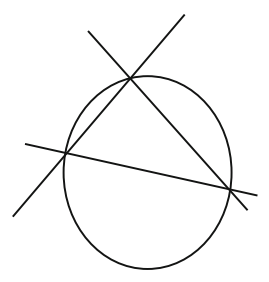

(a)

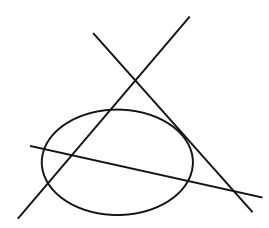

(b)

Fig. 3 CL arrangements with an abelian affine fundamental group $\mathbb{Z}^{4}$ : arrangement $(\mathbf{a})$ has $\beta(\mathcal{A})=1>0$, and arrangement (b) has a tangency point

all the multiple points have multiplicity 3 , the corresponding fundamental group is abelian.

Note also that there are CL arrangements with a tangent point (which is excluded by our restrictions, see Assumption 2.4) with an abelian fundamental group of the complement. For example, three lines and a conic which is tangent only to one of the lines (see Fig. 3b) has an abelian fundamental group (see [8]).

\subsection{The braid monodromy of plane curves and the Zariski-van Kampen theorem}

The reader who is familiar with the definition of the braid monodromy, its computation and the relevant Zariski-van Kampen theorem, can skip this subsection.

We start by defining the braid monodromy associated to a plane curve.

Definition 2.8 Let $D$ be a closed disk in $\mathbb{R}^{2}$, and $K \subset \operatorname{Int}(\mathrm{D})$ a finite set of $n$ points. The braid group $B_{n}[D, K]$ can be defined as the group of equivalence classes of diffeomorphisms $\beta$ of $D$ such that $\beta(K)=K$ and $\left.\beta\right|_{\partial D}=\left.\operatorname{Id}\right|_{\partial D}$, where two diffeomorphisms are equivalent if they induce the same automorphism on $\pi_{1}(D-K, u)$.

Let $a, b \in K$, and let $\sigma$ be a smooth simple path in Int(D) connecting $a$ with $b$ such that $\sigma \cap K=\{a, b\}$. Choose a small regular neighborhood $U$ of $\sigma$ contained in Int(D), such that $U \cap K=\{a, b\}$. The diffeomorphism of $D$ that switches the points $a$ and $b$ by a counterclockwise $180^{\circ}$ rotation and is the identity on $D-U$, defines an element of $B_{n}[D, K]$, called the half-twist defined by $\sigma$ and denoted by $H(\sigma)$.

Definition 2.9 The braid monodromy with respect to $C, \pi, u$.

Let $C \subset \mathbb{C}^{2}$ be a curve. Choose a point $O \in \mathbb{C}^{2}, O \notin C$, such that the projection $p: \mathbb{C}^{2} \rightarrow \mathbb{C}^{1}=\ell$ with a center $O$ (to a generic line $\ell$ called the reference line) will be generic when restricting it to $C$. Denote $\pi=\left.p\right|_{C}$ and let $m=\operatorname{deg} \pi=\operatorname{deg} C$. Let $N=\left\{x \in \ell \mid \# \pi^{-1}(x)<m\right\}$. Take $u \in \ell-N$, and let $\mathbb{C}_{u}^{1}=p^{-1}(u)$. There is a naturally defined homomorphism:

$$
\varphi: \pi_{1}(\ell-N, u) \rightarrow B_{m}\left[\mathbb{C}_{u}^{1}, \mathbb{C}_{u}^{1} \cap C\right]
$$

which is called the braid monodromy with respect to $C, \pi, u$, describing the motion of the points in the fiber (see [22]). 
In fact, letting $E$ be a big disk in $\ell=\mathbb{C}^{1}$ such that $N \subset E$, we can also choose the path in $E-N$ not to be a loop, but just a non-selfintersecting path. This induces a diffeomorphism between the models $(D, K)$ at the two ends of the considered path, where $D$ is a big disk in $\mathbb{C}_{u}^{1}$, and $K=\mathbb{C}_{u}^{1} \cap C \subset D$.

Definition 2.10 $\psi_{T}$, the Lefschetz diffeomorphism induced by a path $T$.

Let $x_{0}, x_{1} \in E-N$ be two different points, $T:[0,1] \rightarrow E-N$ a nonselfintersecting path in $E-N$ connecting $x_{0}$ with $x_{1}$. There exists a continuous family of diffeomorphisms $\psi_{(t)}: D \rightarrow D, t \in[0,1]$, such that $\psi_{(0)}=\mathrm{Id}$, $\psi_{(t)}\left(K\left(x_{0}\right)\right)=K(T(t))$ for all $t \in[0,1]$, and $\psi_{(t)}(y)=y$ for all $y \in \partial D$. For emphasis, we write $\psi_{(t)}:\left(D, K\left(x_{0}\right)\right) \rightarrow(D, K(T(t)))$. The Lefschetz diffeomorphism induced by a path $T$ is the diffeomorphism:

$$
\psi_{T}=\psi_{(1)}:\left(D, K\left(x_{0}\right)\right) \stackrel{\sim}{\rightarrow}\left(D, K\left(x_{1}\right)\right) .
$$

Since $\psi_{(t)}\left(K\left(x_{0}\right)\right)=K(T(t))$ for all $t \in[0,1]$, we have a family of canonical isomorphisms:

$$
\psi_{(t)}^{\nu}: B_{m}\left[D, K\left(x_{0}\right)\right] \stackrel{\sim}{\rightarrow} B_{m}[D, K(T(t))], \quad \text { for all } t \in[0,1]
$$

Let $\left\{\Gamma_{i}\right\}$ be a geometric (free) base (called a g-base) of $\pi_{1}\left(\mathbb{C}^{1}-N, u\right)$ (see [22] for the exact definition), $\varphi$ the braid monodromy of $C, \varphi: \pi_{1}\left(\mathbb{C}^{1}-N, u\right) \rightarrow B_{m}$. In order to find out a presentation of the fundamental group of the complement of $C$ in $\mathbb{C}^{2}$, we have to find out what are $\varphi\left(\Gamma_{i}\right)$, for all $i$. We refer the reader to the definition of a skeleton $\lambda_{x_{j}}$, for all $x_{j} \in N$ (see [23]), which is a model of a set of consecutive paths connecting points in the fiber, which coincide when approaching $A_{j}=\left(x_{j}, y_{j}\right) \in C$ from the right. To describe this situation in more details, for $x_{j} \in N$, let $x_{j}^{\prime}=x_{j}+\alpha$, where $0<\alpha \ll 1$. The skeleton of $x_{j}$ is defined as a system of consecutive paths connecting the points in $K\left(x_{j}^{\prime}\right) \cap D\left(A_{j}, \varepsilon\right)$, where $0<\alpha \ll \varepsilon \ll 1$ and $D\left(A_{j}, \varepsilon\right)$ is a disk centered in $A_{j}$ of radius $\varepsilon$.

For a given skeleton, denote by $\Delta\left\langle\lambda_{x_{j}}\right\rangle$ the braid which rotates a small neighborhood of the given skeleton by $180^{\circ}$ counterclockwise. Note that if $\lambda_{x_{j}}$ is a single path, then $\Delta\left\langle\lambda_{x_{j}}\right\rangle=H\left(\lambda_{x_{j}}\right)$.

We also refer the reader to the definition of $\delta_{x_{0}}$, for $x_{0} \in N$ (see [23]), which describes the Lefschetz diffeomorphism induced by a path going below $x_{0}$, for different types of singular points (either a transversal intersection of several lines at a point or a branch point; for example, when going below a node the corresponding Lefschetz diffeomorphism is a half-twist of the corresponding skeleton).

Thus, the Lefschetz diffeomorphism induced by a path going from $x_{j}^{\prime}$ to $u$ below the points $x_{i}, 1 \leq i \leq j-1$, is the composition of the corresponding $\delta_{x_{i}}$ 's, i.e. $\prod_{m=j-1}^{1} \delta_{x_{m}}[22,23]$. We illustrate the action of a specific Lefschetz diffeomorphism (induced by a line arrangement) in the following example.

Example 2.11 We present here an example for computing a skeleton and the effect of applying a Lefschetz diffeomorphism on it (more examples can be found in $[10,22,23])$. 


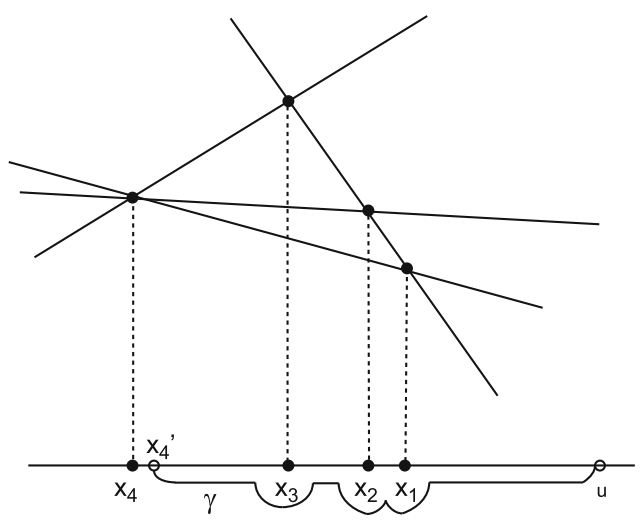

(a)

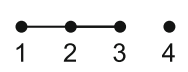

(b)

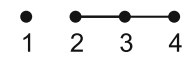

(c)

Fig. 4 Part a is an example of a line arrangement, Part $\mathbf{b}$ is the initial skeleton of the point $x_{4}$ (i.e. in the fiber over $x_{4}^{\prime}$ ) and Part $\mathbf{c}$ is its final skeleton (in the fiber over $u$ )

$\lambda_{x_{4}}$, the initial skeleton of the point $x_{4}$ in Fig. 4a (i.e. in the fiber over $x_{4}^{\prime}$ ), is presented in Fig. 4b.

$\delta$, which is the Lefschetz diffeomorphism induced by $\gamma$ (a path going from $x_{4}^{\prime}$ to $u$ ), is:

$$
\Delta\langle 3,4\rangle \Delta\langle 2,3\rangle \Delta\langle 1,2\rangle
$$

$\lambda_{x_{4}}^{\prime}$, the final skeleton of the point $x_{4}$ in Fig. 4 a (i.e. in the fiber over $u$ after applying $\delta$ on $\lambda_{x_{4}}$ ), is presented in Fig. 4c.

Based on the braid monodromy, we can compute presentations for the groups $\pi_{1}\left(\mathbb{C P}^{2}-\bar{C}\right)$ and $\pi_{1}\left(\mathbb{C}^{2}-C\right)$ (where $\bar{C} \subset \mathbb{C P}^{2}$ is a projective curve, $C=\bar{C} \cap \mathbb{C}^{2}$ ).

Let $\left\{\Gamma_{i}\right\}$ be a $g$-base of $G=\pi_{1}\left(\mathbb{C}_{u}^{1}-\left(\mathbb{C}_{u}^{1} \cap C\right), u\right)$, where $\mathbb{C}_{u}^{1}=\pi^{-1}(u)=\mathbb{C} \times\{u\}$. Then, $\pi_{1}\left(\mathbb{C}^{2}-C, u\right)$ is generated by the images of $\left\{\Gamma_{i}\right\}$ in $\pi_{1}\left(\mathbb{C}^{2}-C, u\right)$. We use now the Zariski-van Kampen theorem [28] in order to compute the relations between the generators of $G$. The theorem essentially says that every singular point (with respect to a projection from $O$ to $\ell$ ) induces a relation in $\pi_{1}\left(\mathbb{C}^{2}-C\right)$, and these induced relations are all the relations of $\pi_{1}\left(\mathbb{C}^{2}-C\right)$.

Since we are dealing only with CL arrangements, we formulate the theorem only for a curve having only branch points, nodes and multiple intersection points as singular points (with respect to a projection).

Theorem 2.12 (Zariski-van Kampen [28]) Let $\bar{C}$ be a $C L$ arrangement in $\mathbb{C P}^{2}$ and $C=\mathbb{C}^{2} \cap \bar{C}$. Let $s$ be the number of singular points of $C$ with respect to the projection from $O$. For every $j \in\{1, \ldots, s\}$, consider the skeleton:

$$
\lambda_{x_{j}}^{\prime}=\left\langle\left(\lambda_{x_{j}}\right)\left(\prod_{m=j-1}^{1} \delta_{x_{m}}\right)\right\rangle .
$$


Then, $\pi_{1}\left(\mathbb{C}^{2}-C, u\right)$ is generated by the images of $\left\{\Gamma_{i}\right\}$ in $\pi_{1}\left(\mathbb{C}^{2}-C, u\right)$ and the only relations are those induced by the skeletons $\lambda_{x_{j}}^{\prime}$ in the following way:

If the point $x_{j}$ is either a node or a branch point, then the skeleton $\lambda_{x_{j}}^{\prime}$ is a path connecting two points. In this case, the relation is either $a_{1} a_{2}=a_{2} a_{1}$ (for a node) or $a_{1}=a_{2}$ (for a branch point), where the computation of the $a_{i}$ 's will be described after the theorem.

If the point $x_{j}$ is an intersection point of multiplicity $k$, then the skeleton $\lambda_{x_{j}}^{\prime}$ is a set of $k-1$ consecutive paths connecting $k$ points. In this case, the relations are:

$$
a_{k} a_{k-1} \cdots a_{1}=a_{1} a_{k} a_{k-1} \cdots a_{2}=\cdots=a_{k-1} a_{k-2} \cdots a_{1} a_{k}
$$

where the computation of the $a_{i}$ 's will be described after the theorem.

Notation 2.13 The relations

$$
a_{k} a_{k-1} \cdots a_{1}=a_{1} a_{k} a_{k-1} \cdots a_{2}=\cdots=a_{k-1} a_{k-2} \cdots a_{1} a_{k}
$$

will be denoted in an abbreviated form as:

$$
\left[a_{1}, a_{2}, \ldots, a_{k}\right]=e
$$

We start by describing the $a_{i}$ 's in the case that the skeleton is a path connecting two points, i.e. the singular point is either a node or a branch point. Let $D$ be a disk circumscribing the skeleton, and let $K$ be the set of points. Choose an arbitrary point on the path and 'pull' it down to $\partial D$, splitting the path into two parts, which are connected at one end to $u_{0} \in \partial D$ and at the other end to the two endpoints of the path in $K$. The loops associated to these two paths are elements in the group $\pi_{1}\left(D-K, u_{0}\right)$ and we call them $a_{1}$ and $a_{2}$. The corresponding elements commute (in the case of a node) or equal (in the case of a branch point) in the fundamental group of the arrangement's complement. Figure 5 illustrates this procedure.

Now we show how to write $a_{1}$ and $a_{2}$ as words in the generators $\left\{\Gamma_{1}, \ldots, \Gamma_{\ell}\right\}$ of $\pi_{1}\left(D-K, u_{0}\right)$. We start with the generator corresponding to the endpoint of $a_{1}$ (or $a_{2}$ ), and conjugate it as we move along $a_{1}$ (or $a_{2}$ ) from its endpoint in $K$ to $u_{0}$ as follows: for every point $i \in K$ which we pass from above, we conjugate by $\Gamma_{i}$ while moving from left to right, and by $\Gamma_{i}^{-1}$ while moving from right to left.

For example, in Fig. 5,

$$
a_{1}=\Gamma_{3} \Gamma_{2} \Gamma_{1} \Gamma_{2}^{-1} \Gamma_{3}^{-1}, \quad a_{2}=\Gamma_{4}^{-1} \Gamma_{6} \Gamma_{4}
$$

Assuming that the singular point is a node, the induced relation is the following commutative relation:

$$
\Gamma_{3} \Gamma_{2} \Gamma_{1} \Gamma_{2}^{-1} \Gamma_{3}^{-1} \cdot \Gamma_{4}^{-1} \Gamma_{6} \Gamma_{4}=\Gamma_{4}^{-1} \Gamma_{6} \Gamma_{4} \cdot \Gamma_{3} \Gamma_{2} \Gamma_{1} \Gamma_{2}^{-1} \Gamma_{3}^{-1} .
$$

One can check that the induced relation is independent of the point in which the path is split. 

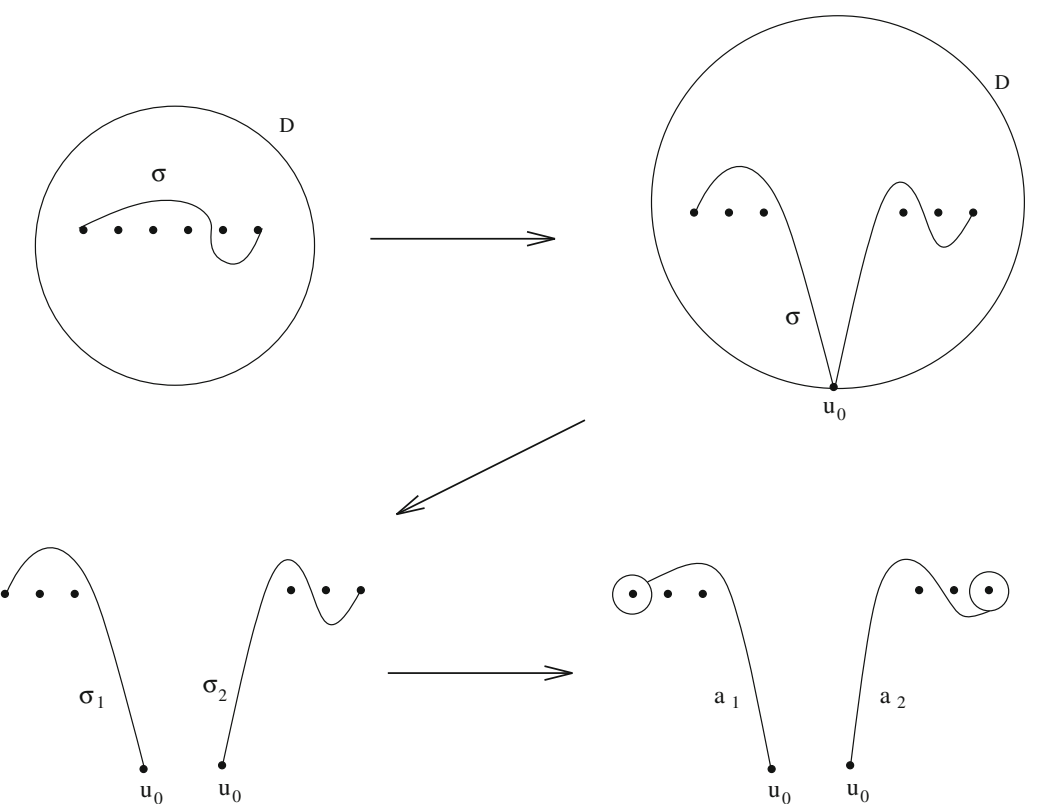

Fig. 5 Construction of $a_{1}, a_{2}$ for a node or a branch point

For an intersection point of multiplicity $k$, we compute the elements in the group $\pi_{1}\left(D-K, u_{0}\right)$ in a similar way, but the induced relations are of the following cyclic type:

$$
a_{k} a_{k-1} \cdots a_{1}=a_{1} a_{k} a_{k-1} \cdots a_{2}=\cdots=a_{k-1} a_{k-2} \cdots a_{1} a_{k} .
$$

For computing the $a_{i}$ 's, we choose an arbitrary point on one of the paths and pull it down to $u_{0}$. For each of the $k$ points of the skeleton, we generate the loop associated to the path from $u_{0}$ to that point, and translate this path to a word in $\Gamma_{1}, \ldots, \Gamma_{\ell}$ by the procedure described above (note that while computing the word corresponding to $a_{j}$, the path $a_{j}$ is considered as being below the final points of $a_{i}, i \neq j$ ).

In the example given in Fig. 6, we have:

$$
a_{1}=\Gamma_{3} \Gamma_{1} \Gamma_{3}^{-1}, a_{2}=\Gamma_{3} \Gamma_{2} \Gamma_{3}^{-1} \text { and } a_{3}=\Gamma_{4}^{-1} \Gamma_{6} \Gamma_{4},
$$

so the induced relations are:

$$
\begin{aligned}
\Gamma_{4}^{-1} \Gamma_{6} \Gamma_{4} \cdot \Gamma_{3} \Gamma_{2} \Gamma_{3}^{-1} \cdot \Gamma_{3} \Gamma_{1} \Gamma_{3}^{-1} & =\Gamma_{3} \Gamma_{1} \Gamma_{3}^{-1} \cdot \Gamma_{4}^{-1} \Gamma_{6} \Gamma_{4} \cdot \Gamma_{3} \Gamma_{2} \Gamma_{3}^{-1} \\
& =\Gamma_{3} \Gamma_{2} \Gamma_{3}^{-1} \cdot \Gamma_{3} \Gamma_{1} \Gamma_{3}^{-1} \cdot \Gamma_{4}^{-1} \Gamma_{6} \Gamma_{4}
\end{aligned}
$$



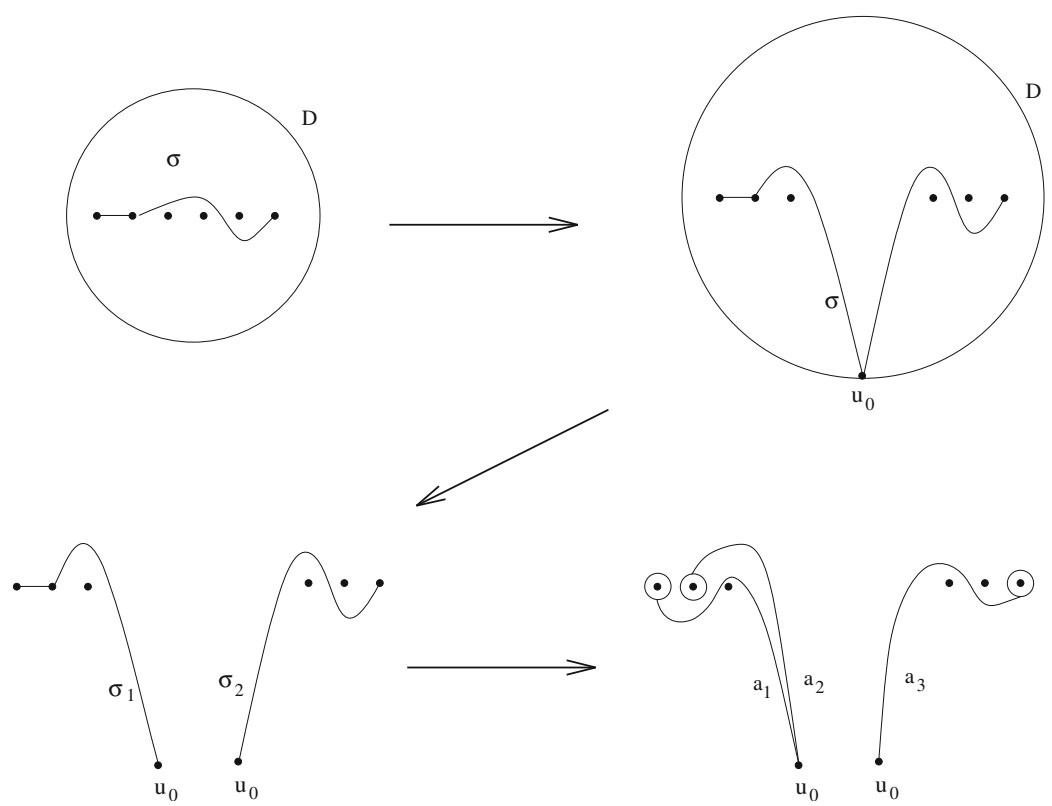

Fig. 6 Construction of $a_{1}, a_{2}, a_{3}$ for a multiple intersection point

\subsection{The conjugation-free property}

In this section, we define the notion of a conjugation-free geometric presentation for the fundamental group of line and CL arrangements, following the definition given in [10]:

Definition 2.14 Let $G$ be a fundamental group of the affine or projective complements of a real CL arrangement with $k$ lines and $n$ conics (where $k>0$ and $n \geq 0$ ). We say that $G$ has a conjugation-free geometric presentation if $G$ has a presentation with the following properties:

- In the affine case, the generators $\left\{x_{1}, \ldots, x_{k+2 n}\right\}$ are the meridians of lines and conics at $\mathbb{C}_{u}^{1}=\pi^{-1}(u)$, and therefore there are $k+2 n$ generators.

- In the projective case, the generators are the meridians of lines and conics at $\mathbb{C}_{u}^{1}=\pi^{-1}(u)$ except for one, and therefore there are $k+2 n-1$ generators.

- In both cases, the induced relations are of the following types:

$$
x_{i_{t}} x_{i_{t-1}} \cdots x_{i_{1}}=x_{i_{t-1}} \cdots x_{i_{1}} x_{i_{t}}=\cdots=x_{i_{1}} x_{i_{t}} \cdots x_{i_{2}}
$$

induced by an intersection point of multiplicity $t$, or

$$
x_{i_{1}}=x_{i_{2}}
$$


induced by a branch point, where $\left\{i_{1}, i_{2}, \ldots, i_{t}\right\} \subseteq\{1, \ldots, m\}$ is an increasing subsequence of indices, where $m=k+2 n$ in the affine case and $m=k+2 n-1$ in the projective case. Note that if $t=2$ in the first type, we get the usual commutator.

- In the projective case, we have an extra relation that a specific multiplication of all the generators is equal to the identity element.

Note that in each case we claim that with respect to particular choices of the reference line $\ell$ (i.e. the line to which we project the arrangement), the point $u$ (the basepoint for both the meridians in the fiber $\mathbb{C}_{u}^{1}$ and the loops in the group $\pi_{1}(\ell-N, u)$ ) and the projection point $O$, we have this conjugation-free property.

Remark 2.15 In the model we work with, the reference line $\ell$ is $\ell=\{y=a\}, a \ll 0$, where $\ell$ is chosen to be below all the real singular points of the arrangement, the projection in $\mathbb{C}^{2}$ is $(x, y) \rightarrow x$ (i.e. in $\mathbb{C P}^{2}$, the point $O$ is $\left.(1: 0: 0)\right)$ and the point $u \in \ell$ is always a real point, i.e. $u=\left(u_{x}, a\right)$, where $u_{x} \in \mathbb{R}$.

As will be proven below, for certain families of line arrangements the property of having a conjugation-free geometric presentation is independent of the choice of $u$. We thus conjecture the following:

Conjecture 2.16 The property of having a conjugation-free geometric presentation is independent of the choices of $O, \ell$ and $u \in \ell$.

Remark 2.17 The notion of a conjugation-free geometric presentation for the fundamental group can be generalized to any arrangement of plane curves (with the proper modifications with respect to the degrees of the curves and the types of singularities).

Note that the importance of the family of CL arrangements whose fundamental group has a conjugation-free geometric presentation is that such a presentation of the fundamental group can be read directly from the arrangement without any additional computation, and thus depends on the combinatorics of the arrangement.

The goal of the next two sections is to give a complete proof to the following proposition:

Proposition 2.18 (1) Let $\mathcal{L}$ be a real line arrangement such that $\pi_{1}\left(\mathbb{C}^{2}-\mathcal{L}, u\right)$ has a conjugation-free geometric presentation for any real basepoint $u \in \ell-N$ (where $N$ is the set of the projection of singular points with respect to the projection $\pi$ ). Let $L$ be a real line not in $\mathcal{L}$ that passes through a single intersection point of $\mathcal{L}$. Then $\pi_{1}\left(\mathbb{C}^{2}-(\mathcal{L} \cup L), u\right)$ has a conjugation-free geometric presentation for any real basepoint $u$.

(2) Let $\mathcal{A}$ be a real CL arrangement with one conic such that $\pi_{1}\left(\mathbb{C}^{2}-\mathcal{A}, u\right)$ has a conjugation-free geometric presentation for any real basepoint $u \in \ell-N$. Let $L$ be a real line not in $\mathcal{A}$ that passes through a single intersection point of $\mathcal{A}$ such that $\beta(\mathcal{A} \cup L)=0$. Then $\pi_{1}\left(\mathbb{C}^{2}-(\mathcal{A} \cup L), u\right)$ has a conjugation-free geometric presentation for any real basepoint $u$.

Although a proof to Proposition 2.18(1) was already given in [11, Proposition 2.2], it is not a complete proof: the authors of [11] assume implicitly that certain operations 
(such as a rotation of the arrangement, moving a singularity through "infinity" or a rotation of a line in the arrangement), that are performed on a line arrangement $\mathcal{L}$, preserve the conjugation-free property. Indeed, while these operations induce isomorphic fundamental groups (as partially proved in [18]), one still has to prove that if $\beta$ is such an operation and if $\pi_{1}\left(\mathbb{C}^{2}-\mathcal{L}\right)$ has a conjugation-free geometric presentation with respect to the indicated model above (see Definition 2.14 and Remark 2.15), then $\pi_{1}\left(\mathbb{C}^{2}-\beta(\mathcal{L})\right)$ has also a conjugation-free geometric presentation with respect to the same model.

\section{Preservation of conjugation-freeness: the case of real line arrangements}

The goal of this section is to give a complete proof to Proposition 2.18(1) (which corrects the proof given in [11]): given a real line arrangement $\mathcal{L}$ whose fundamental group has a conjugation-free presentation, then, under certain conditions, passing a real line $L$ through at most one singular point of $\mathcal{L}$, the new arrangement $\mathcal{L} \cup L$ has the same property of conjugation-freeness. We divide the proof into a sequence of lemmata, as we have to prove that the following operations preserve the conjugation-free property:

- $h_{1}$ : given a line $\ell \in \mathcal{L}$ that passes through a single intersection point $p \in \mathcal{L}$, rotate the line around $p$ as long as it does not coincide with a different line.

- $h_{2}$ : given a line $\ell \in \mathcal{L}$ that passes through a single intersection point $p \in \mathcal{L}$, move the line over a different line $\ell^{\prime}$ that passes also through $p$ and through another multiple point (i.e. the deformation takes place in $\mathbb{C}^{2}$, see Fig. 7).

- $h_{3}$ : Changing the basepoint $u \in \ell-N$ when computing $\pi_{1}\left(\mathbb{C}^{2}-\mathcal{L}, u\right)$ (where $N$ is defined in Definition 2.9).

Having all these operations preserving the conjugation-free property, we can indeed claim (as was claimed in the proof of [11, Proposition 2.2]) that after adding the new line, we still have that the fundamental group of the new arrangement has a conjugationfree geometric presentation.

Remark 3.1 (1) Comparing to [11], we reduced the number of operations (performed on $\mathcal{L}$ ) preserving the conjugation-free property. We are not proving that a rotation (of the whole arrangement) or the moving of a singular point through "infinity" preserve the conjugation-free property; instead of these operations, we only prove that changing the real basepoint $u \in \ell-N$ preserves this property. However,

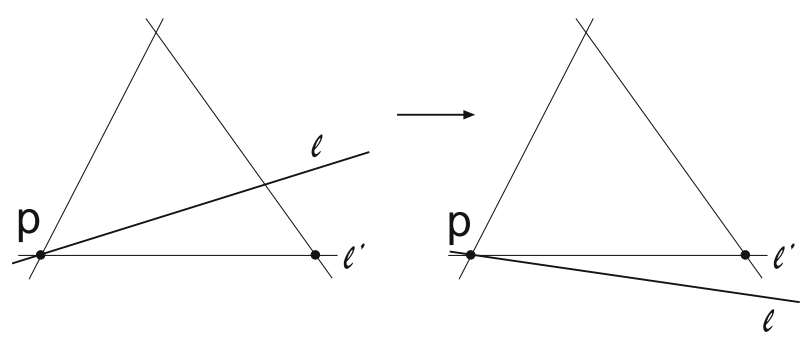

Fig. 7 Moving a line $\ell$ over another line $\ell^{\prime}$ 
Lemma 6.8 below deals with the preservation of the conjugation-free property during rotating certain classes of line arrangements.

(2) Note also that it is not clear if the operation of moving a singular point through "infinity", that was presented in [18] in the context of wiring diagrams, can be applied for line arrangements. Indeed, if one of the lines that passes through an intersection point $p$, passes also through many other multiple intersection points, it is not clear that one can move $p$ through "infinity" without changing the intersection lattice with respect to this line.

Thus, given a real line arrangement $\mathcal{L}$, we have to answer the following two questions: first, what is the explicit isomorphism $f_{i}: \pi_{1}\left(\mathbb{C}^{2}-\mathcal{L}\right) \rightarrow \pi_{1}\left(\mathbb{C}^{2}-h_{i}(\mathcal{L})\right)$ for $1 \leq i \leq 3$ ? and second, does this isomorphism preserve the conjugation-free property?

Remark 3.2 Note that from now on, whenever we refer to the basepoint $u \in \ell$, it is implicitly assumed that $u$ is real.

Lemma 3.3 The operation $h_{1}$ preserves the conjugation-free property.

Proof Let $\mathcal{L}$ be a real line arrangement. The operation $h_{1}$ - rotating a line over a multiple point - and its effects on the fundamental group is described in [18, Section 4.5]. In [18, Theorem 4.13] it is proven that the isomorphism $f_{1}: \pi_{1}\left(\mathbb{C}^{2}-\mathcal{L}, u\right) \rightarrow$ $\pi_{1}\left(\mathbb{C}^{2}-h_{1}(\mathcal{L}), u\right)$ is given by $\Gamma_{j} \mapsto \Gamma_{j}^{\prime}$, where $\Gamma_{j}$ (resp. $\Gamma_{j}^{\prime}$ ) are the geometric generators for $\pi_{1}\left(\mathbb{C}^{2}-\mathcal{L}\right)$ (resp. $\left.\pi_{1}\left(\mathbb{C}^{2}-h_{1}(\mathcal{L})\right)\right)$ and $u \in \ell-N$ is a basepoint located to the right of all the points in $N \subset \ell$. Since $f_{1}$ sends generators to generators, it is obvious that the relations in $\pi_{1}\left(\mathbb{C}^{2}-h_{1}(\mathcal{L})\right)$ have no conjugations, and hence the new presentation is still conjugation-free.

Lemma 3.4 The operation $h_{2}$ preserves the conjugation-free property.

Proof Let $\mathcal{L}$ be a real arrangement of $n$ lines, $\ell_{b} \in \mathcal{L}$ a line that passes through a single multiple point $p \in \mathcal{L}$ and $\ell_{a} \in \mathcal{L}$ a different line that passes through $p$ and through another multiple point $p^{\prime}$, see Fig. 8 a. Note that $a, b \in\{1, \ldots, n\}$ are the numerations of the lines in the fiber over the basepoint $u$.

First, by Lemma 3.3, we can rotate the line $\ell_{b}$ (where the center of rotation is $p$ ) till it is very close to $p^{\prime}$, not coinciding with $\ell_{a}$, see Fig. 8 b. Assuming that $x(p) \ll u$, we note that while numerating the points in the fiber over $u, b=a+1$. Then, we rotate the line $\ell_{b}$ over $\ell_{a}$, such that in the fiber $\mathbb{C}_{u}^{1}$, the corresponding point $p_{b}$ will perform a $180^{\circ}$ clockwise rotation and the point $p_{a}$ will remain unchanged (see Fig. $8 \mathrm{c}$ ).

The induced isomorphism $f_{2}: \pi_{1}\left(\mathbb{C}^{2}-\mathcal{L}, u\right) \rightarrow \pi_{1}\left(\mathbb{C}^{2}-h_{2}(\mathcal{L}), u\right)$ is given by the following map:

$$
\begin{gathered}
\Gamma_{b} \mapsto \Gamma_{a}^{\prime}, \\
\Gamma_{a} \mapsto \Gamma_{a}^{\prime-1} \Gamma_{b}^{\prime} \Gamma_{a}^{\prime}, \\
\Gamma_{i} \mapsto \Gamma_{i}^{\prime}, \quad i \neq a, b,
\end{gathered}
$$




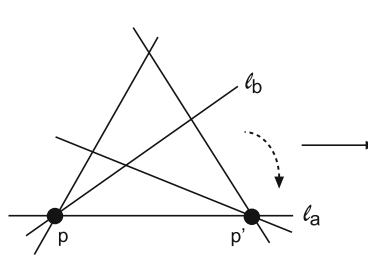

(a)

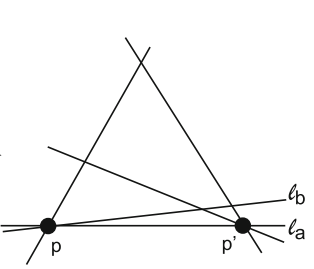

(b)
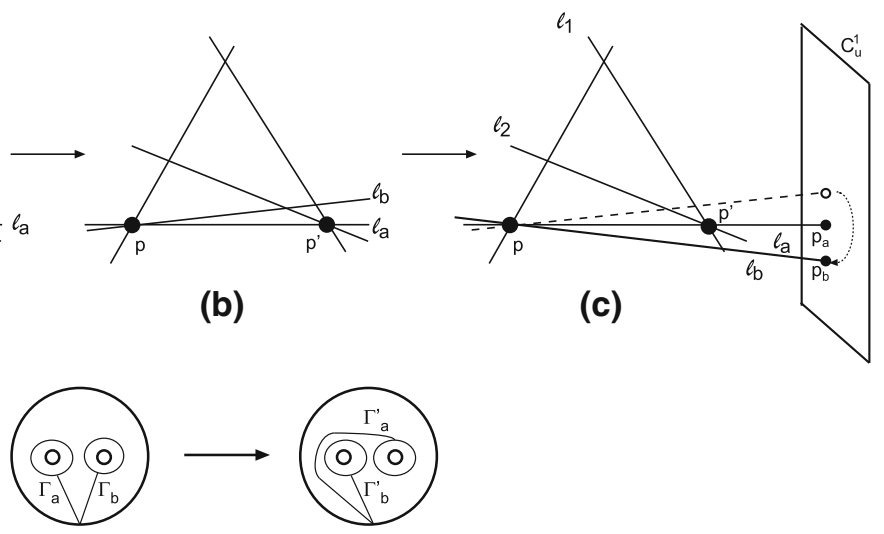

(d)

Fig. 8 The rotation of the line $\ell_{b}$ over another line $\ell_{a}(\mathbf{a}-\mathbf{c})$ and its effect on the geometric generators $(\mathbf{d})$

(see Fig. 8d). Note that the only relations in $\pi_{1}\left(\mathbb{C}^{2}-h_{2}(\mathcal{L}), u\right)$ that presumably have conjugations are those that involved the generator $\Gamma_{a}$ in $\pi_{1}\left(\mathbb{C}^{2}-\mathcal{L}, u\right)$, i.e. the relations induced by the intersection points that are on $\ell_{a}$.

The relation induced by the point $p$ in $\pi_{1}\left(\mathbb{C}^{2}-\mathcal{L}, u\right)$ is:

$$
\left[\Gamma_{i_{1}}, \ldots, \Gamma_{i_{m}}, \Gamma_{a}, \Gamma_{b}, \Gamma_{i_{m+3}}, \ldots, \Gamma_{i_{k}}\right]=e
$$

(where $k$ is the multiplicity of $p$ ), which is mapped in $\pi_{1}\left(\mathbb{C}^{2}-h_{2}(\mathcal{L}), u\right)$ to:

$$
\left[\Gamma_{i_{1}}^{\prime}, \ldots, \Gamma_{i_{m}}^{\prime}, \Gamma_{a}^{\prime-1} \Gamma_{b}^{\prime} \Gamma_{a}^{\prime}, \Gamma_{a}^{\prime}, \Gamma_{i_{m+3}}^{\prime}, \ldots, \Gamma_{i_{k}}^{\prime}\right]=e .
$$

Now, after the rotation of the line $\ell_{b}$, the numeration of the lines passing through $p$ is as follows:

$$
i_{1}, \ldots, i_{m}, a, b=a+1, i_{m+3}, \ldots, i_{k},
$$

which means that we need to prove that the following relation holds in $\pi_{1}\left(\mathbb{C}^{2}-h_{2}(\mathcal{L}), u\right)$ :

$$
\left[\Gamma_{i_{1}}^{\prime}, \ldots, \Gamma_{i_{m}}^{\prime}, \Gamma_{a}^{\prime}, \Gamma_{b}^{\prime}, \Gamma_{i_{m+3}}^{\prime}, \ldots, \Gamma_{i_{k}}^{\prime}\right]=e .
$$

However, an easy check (i.e. expanding the brackets of relation (2)) shows that this is indeed the relation we get from relation (1). For example, for $k=3$, we have that

$$
\left[\Gamma_{i_{1}}, \Gamma_{a}, \Gamma_{b}\right]=e \rightarrow\left[\Gamma_{i_{1}}^{\prime}, \Gamma_{a}^{\prime-1} \Gamma_{b}^{\prime} \Gamma_{a}^{\prime}, \Gamma_{a}^{\prime}\right]=e .
$$

Therefore, the right hand side is equivalent to the following relations:

$$
\begin{aligned}
& \Gamma_{a}^{\prime-1} \Gamma_{b}^{\prime} \Gamma_{a}^{\prime} \cdot \Gamma_{i_{1}}^{\prime} \cdot \Gamma_{a}^{\prime}=\Gamma_{i_{1}}^{\prime} \cdot \Gamma_{a}^{\prime} \cdot \Gamma_{a}^{\prime-1} \Gamma_{b}^{\prime} \Gamma_{a}^{\prime} \\
& \quad \Rightarrow \Gamma_{b}^{\prime} \Gamma_{a}^{\prime} \Gamma_{i_{1}}^{\prime}=\Gamma_{a}^{\prime} \Gamma_{i_{1}}^{\prime} \Gamma_{b}^{\prime}
\end{aligned}
$$


and

$$
\begin{gathered}
\Gamma_{a}^{\prime} \cdot \Gamma_{a}^{\prime-1} \Gamma_{b}^{\prime} \Gamma_{a}^{\prime} \cdot \Gamma_{i_{1}}^{\prime}=\Gamma_{a}^{\prime-1} \Gamma_{b}^{\prime} \Gamma_{a}^{\prime} \cdot \Gamma_{i_{1}}^{\prime} \cdot \Gamma_{a}^{\prime} \Rightarrow \Gamma_{a}^{\prime} \Gamma_{b}^{\prime} \Gamma_{a}^{\prime} \Gamma_{i_{1}}^{\prime}=\Gamma_{b}^{\prime} \Gamma_{a}^{\prime} \Gamma_{i_{1}}^{\prime} \Gamma_{a}^{\prime} \\
\stackrel{\text { Eqn.(3) }}{\Rightarrow} \Gamma_{a}^{\prime} \Gamma_{b}^{\prime} \Gamma_{a}^{\prime} \Gamma_{i_{1}}^{\prime}=\Gamma_{a}^{\prime} \Gamma_{i_{1}}^{\prime} \Gamma_{b}^{\prime} \Gamma_{a}^{\prime} \Rightarrow \Gamma_{b}^{\prime} \Gamma_{a}^{\prime} \Gamma_{i_{1}}^{\prime}=\Gamma_{i_{1}}^{\prime} \Gamma_{b}^{\prime} \Gamma_{a}^{\prime} .
\end{gathered}
$$

Thus, relations (3) and (4) imply that $\left[\Gamma_{i_{1}}^{\prime}, \Gamma_{a}^{\prime}, \Gamma_{b}^{\prime}\right]=e$.

Let $p^{\prime}$ be another intersection point on $\ell_{a}$ (see Fig. 8c). Assume for simplicity that only two more lines $\ell_{1}, \ell_{2}$ pass through $p^{\prime}$ and that the relation induced by $p^{\prime}$ in $\pi_{1}\left(\mathbb{C}^{2}-\mathcal{L}, u\right)$ is $\left[\Gamma_{1}, \Gamma_{a}, \Gamma_{2}\right]=e$. As $\ell_{b}$ passes through only one multiple point $p$, it intersects $\ell_{1}, \ell_{2}$ transversally, and therefore the induced relations in $\pi_{1}\left(\mathbb{C}^{2}-\mathcal{L}, u\right)$ (which do not have conjugations since the presentation is conjugationfree) are $\left[\Gamma_{1}, \Gamma_{b}\right]=\left[\Gamma_{2}, \Gamma_{b}\right]=e$. Thus, in $\pi_{1}\left(\mathbb{C}^{2}-h_{2}(\mathcal{L}), u\right)$, the corresponding induced relations are:

$$
\left[\Gamma_{1}^{\prime}, \Gamma_{a}^{\prime}\right]=\left[\Gamma_{2}^{\prime}, \Gamma_{a}^{\prime}\right]=e
$$

and hence:

$$
\left[\Gamma_{1}^{\prime}, \Gamma_{a}^{\prime-1} \Gamma_{b}^{\prime} \Gamma_{a}^{\prime}, \Gamma_{2}^{\prime}\right]=e^{\left[\Gamma_{1}^{\prime}, \Gamma_{a}^{\prime}\right]=\left[\Gamma_{2}^{\prime}, \Gamma_{a}^{\prime}\right]=e}\left[\Gamma_{1}^{\prime}, \Gamma_{b}^{\prime}, \Gamma_{2}^{\prime}\right]=e
$$

Therefore, the relation induced by $p^{\prime}$ has no conjugations; hence the new presentation is also conjugation-free, as needed.

Note that the last part of the proof is independent of the order of the generators (i.e. how the lines $\ell_{1}, \ell_{2}$ pass through $p^{\prime}$ ) and is similar when either only one line passes through the point $p^{\prime}$ or more than three lines pass through this point.

We now treat the operation $h_{3}$. We start with a remark.

Remark 3.5 (I) Let $p$ be an intersection point of $\mathcal{L}$. Changing the basepoint from $u=x(p)+\varepsilon$, where $0<\varepsilon \ll 1$, to $u^{\prime}=x(p)-\varepsilon$ (see Fig. 9) induces an isomorphism on the geometric generators, which is described below.

If the local skeleton at the point $p$ is composed of the lines numerated by $a, a+$ $1, \ldots, a+s$, and the geometric generators of the group $\pi_{1}\left(\mathbb{C}^{2}-\mathcal{L}, u\right)$ (resp. $\left.\pi_{1}\left(\mathbb{C}^{2}-\mathcal{L}, u^{\prime}\right)\right)$ are $\Gamma_{i}\left(\right.$ resp. $\left.\Gamma_{i}^{\prime}\right)$, then the isomorphism $f_{3}: \pi_{1}\left(\mathbb{C}^{2}-\mathcal{L}, u\right) \rightarrow$ $\pi_{1}\left(\mathbb{C}^{2}-\mathcal{L}, u^{\prime}\right)$ is given by:

$$
f_{3}\left(\Gamma_{i}\right)=\left\{\begin{array}{cl}
\Gamma_{i}^{\prime} & 1 \leq i<a \\
\Gamma_{a}^{\prime-1} \cdots \Gamma_{a+s-1}^{\prime-1} \Gamma_{a+s}^{\prime} \Gamma_{a+s-1}^{\prime} \cdots \Gamma_{a}^{\prime} & i=a \\
\Gamma_{a}^{\prime}-1 \ldots \Gamma_{a+s-2}^{\prime-1} \Gamma_{a+s-1}^{\prime} \Gamma_{a+s-2}^{\prime} \cdots \Gamma_{a}^{\prime} & i=a+1 \\
\vdots & \vdots \\
\Gamma_{a}^{\prime-1} \Gamma_{a+1}^{\prime} \Gamma_{a}^{\prime} & i=a+s-1 \\
\Gamma_{a}^{\prime} & i=a+s \\
\Gamma_{i}^{\prime} & a+s<i \leq n
\end{array}\right.
$$




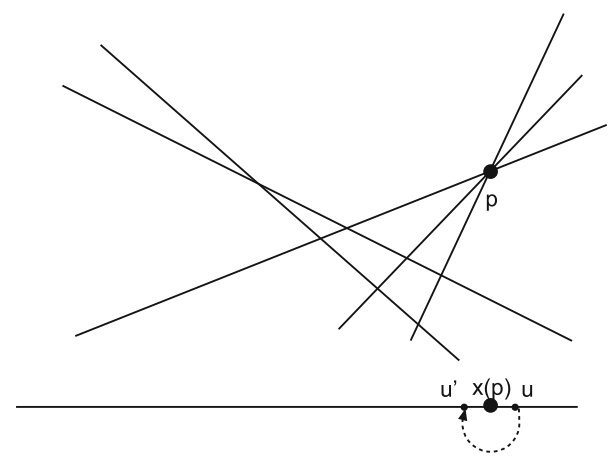

Fig. 9 Changing the location of the basepoint from the right of an intersection point to its left

(II) By the isomorphism presented in (5), one can show that if $p$ is a node, then $f_{3}$ indeed preserves the conjugation-free property. Indeed, if the basepoint is $u^{\prime}$, the relation $\left[\Gamma_{a}^{\prime}, \Gamma_{a+1}^{\prime}\right]=e$ exists. In this case, the isomorphism $f_{3}$ is

$$
\begin{gathered}
\Gamma_{a} \mapsto \Gamma_{a+1}^{\prime}, \\
\Gamma_{a+1} \mapsto \Gamma_{a+1}^{\prime-1} \Gamma_{a}^{\prime} \Gamma_{a+1}^{\prime}\left[\Gamma_{a}^{\prime}, \Gamma_{a+1}^{\prime}\right]=e \\
\Gamma_{a}^{\prime}
\end{gathered}
$$

and since any geometric generator is sent to a geometric generator, $f_{3}$ preserves the conjugation-free property.

Lemma 3.6 Let $\mathcal{L}$ be a real line arrangement which can be presented as a union $\mathcal{L}=\mathcal{L}^{\prime} \cup L$, where $\mathcal{L}^{\prime}$ is a real line arrangement whose fundamental group has a conjugation-free geometric presentation for every real basepoint $u \in \ell-N$ and $L \notin \mathcal{L}^{\prime}$ is a real line that passes through at most one intersection point $p$ of $\mathcal{L}^{\prime}$. Then, on this class of real line arrangements, the operation $h_{3}$ preserves the conjugation-free property for $\mathcal{L}$; that is, the fundamental group $\pi_{1}\left(\mathbb{C}^{2}-\mathcal{L}, u\right)$ has the conjugation-free property for every real basepoint $u \in \ell-N$.

Remark 3.7 Though the condition on $\mathcal{L}$ in Lemma 3.6 seems restrictive, one should note that if an arrangement $\mathcal{L}$ can be built by adding one line that passes through at most one intersection point at each step of the construction, then $\mathcal{L}$ has eventually a conjugation-free geometric presentation, independent of the chosen real basepoint $u$ (such an arrangement has a conjugation-free graph, see Sect. 6 for more details). Indeed, note that for the arrangement $\mathcal{L}_{0}$ that has only nodes, $\pi_{1}\left(\mathbb{C}^{2}-\mathcal{L}_{0}, u\right)$ is abelian (for any real basepoint $u$ ) and therefore every relation has no conjugations, which means that it is independent of the basepoint $u$. Then, one can build $\mathcal{L}$ from $\mathcal{L}_{0}$ by adding only one line at each step (for an exact definition of $\mathcal{L}_{0}$ and an example of this process see the proof of Proposition 6.2 and Sect. 6.1). Thus, by Lemma 3.6, we prove that $\pi_{1}\left(\mathbb{C}^{2}-\mathcal{L}, u\right)$ has a conjugation-free geometric presentation, independent of the location of the real basepoint $u$ on $\ell$. 
Proof We remind again that whenever we refer to the basepoint $u$, we implicitly assume that $u \in \mathbb{C}^{2}$ is real (in its two coordinates). Moreover, since we choose the reference line $\ell$ as $\ell=\{y=a: a \in \mathbb{R}\}, m \ll 0$, the $y$-coordinate of $u$ is fixed. Therefore, when we write, for example, $u=x(m)+\varepsilon$, we mean that $x(u)=x(m)+$ $\varepsilon$, for $m \in \mathbb{R}^{2}$. For the convenience of the reader, we omit the notation $x(u)$ and write only $u$.

First, note that our assumption implies that for every basepoint $u_{0} \in \ell-N$, $\pi_{1}\left(\mathbb{C}^{2}-\mathcal{L}^{\prime}, u_{0}\right)$ has a conjugation-free geometric presentation.

If $L$ intersects every line of $\mathcal{L}^{\prime}$ transversally, then, if

$$
\pi_{1}\left(\mathbb{C}^{2}-\mathcal{L}^{\prime}, u_{0}\right)=\left\langle x_{1}, \ldots, x_{k}: R\right\rangle
$$

is a conjugation-free presentation (where $R$ is a complete set of relations, which have no conjugations), then, denoting by $z$ the generator corresponds to $L$ we have by [25] that:

$$
\begin{aligned}
\pi_{1}\left(\mathbb{C}^{2}-\mathcal{L}, u_{0}\right) & =\mathbb{Z} \oplus \pi_{1}\left(\mathbb{C}^{2}-\mathcal{L}^{\prime}, u_{0}\right) \\
& =\left\langle z, x_{1}, \ldots, x_{k}: R,\left[z, x_{i}\right]=e, 1 \leq i \leq k\right\rangle,
\end{aligned}
$$

which is obviously also a conjugation-free presentation too.

Assume thus that $L$ does not intersect every line of $\mathcal{L}^{\prime}$ transversally. Let $p$ be an intersection point of $\mathcal{L}^{\prime}$. We draw $\mathcal{L}^{\prime}$ through $p$ and assume that $L$ is almost vertical to the reference line $\ell$ with a very negative slope. We can assume that, since after we prove that the arrangement $\mathcal{L}^{\prime} \cup L$ has a conjugation-free geometric presentation, we can use Lemma 3.3 to rotate the line $L$ around the point $p$ (back to its "original" place). The arrangement is now at the position described in Fig. 10a.

We introduce a few notations. Let $p^{\prime}$ be the highest intersection point on $L$. Numerate the intersection points on $L$, starting from the lowest point on $L$, by $p_{0}, p_{1}, \ldots, p_{v}=p, \ldots, p_{u}=p^{\prime}$ (note that all the points $p_{i}$ are nodes, except for $p_{v}$ which is an intersection point of multiplicity $m>2$ ) and denote their corresponding Lefschetz pairs by

- $s_{0}=[1,2], s_{1}=[2,3], \ldots, s_{v-1}=[v, v+1]$,

- $s_{v}=[v+1, v+m]$,

- $s_{v+1}=[v+m, v+m+1], \ldots, s_{u}=[n-1, n]$,

where $n$ is the number of lines in $\mathcal{L}$.

We now divide the proof into a number of steps, each concentrating in a different domain, depending on the location of the basepoint. First, we prove that for the basepoint $u \in \ell$, where $u=x\left(p^{\prime}\right)-\varepsilon, 0<\varepsilon \ll 1$ (see Fig. 10b), $\pi_{1}\left(\mathbb{C}^{2}-\mathcal{L}, u\right.$ ) has a conjugation-free geometric presentation.

Step 3.8 For $u=x\left(p^{\prime}\right)-\varepsilon, \pi_{1}\left(\mathbb{C}^{2}-\mathcal{L}, u\right)$ has a conjugation-free geometric presentation.

Proof We prove this step in two parts. In Part (I), we prove that all the skeletons of the intersection points to the left of $u$ or to the right of $u^{\prime} \doteq x\left(p_{0}\right)+\varepsilon$ are exactly 


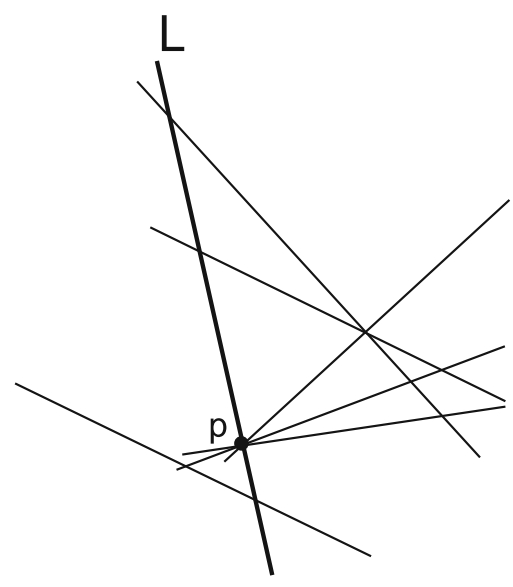

(a)

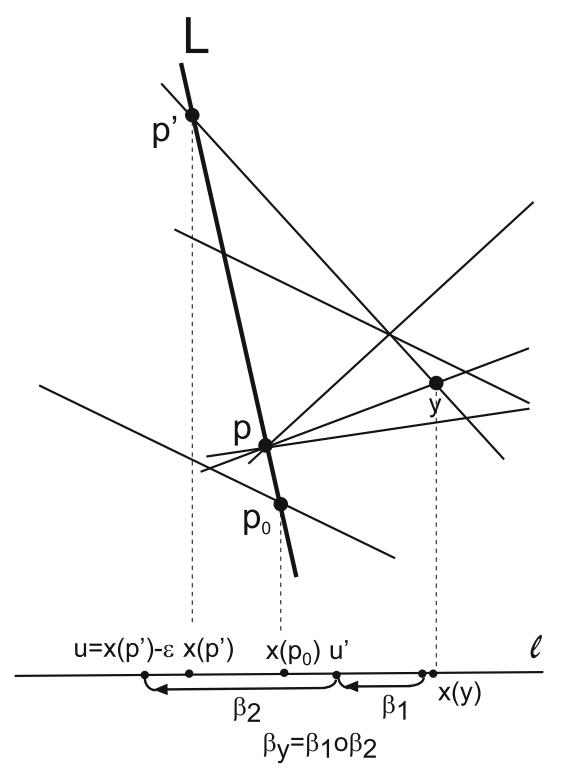

(b)

Fig. 10 Part a describes the setting for adding the line $L$ in the case of real line arrangements. Part b depicts the notations for the proof of the conjugation-free property for Step 3.8

the same when computed in $\mathcal{L}$ or in $\mathcal{L}^{\prime}$. Once we prove that and we prove also that all the relations induced by the intersection points on $L$ have no conjugations, then we can use the fact that $\pi_{1}\left(\mathbb{C}^{2}-\mathcal{L}^{\prime}, u\right)$ is conjugation-free for proving, in Part (II), that $\pi_{1}\left(\mathbb{C}^{2}-\mathcal{L}, u\right)$ has a conjugation-free geometric presentation.

Part (I): Choosing the basepoint to be $u$ yields almost immediately that all the relations induced by intersection points on $L$ have no conjugations. As for the intersection points to the left of $u$, since the intersection points on $L$ are not affecting any of these intersection points, we can consider the resulting skeletons as skeletons of $\mathcal{L}^{\prime}$ (where the only difference is that the fiber contains now an additional point, corresponding to the line $L$, being the highest point in the fiber and not participating in the skeletons). We now have to treat the relations induced by the intersection points which are to the right of $L$.

For every intersection point $y \in \mathcal{L}$ which is to the right of $u^{\prime}$, let $s_{y}$ be the initial skeleton of $y$. We now consider the braids which act on $s_{y}$ while going along a path $\beta_{y}$ starting at $x(y)-\varepsilon$ and ending at $u$. This path can be divided into two parts: the first part $\beta_{1}$ which starts at $x(y)-\varepsilon$ and ends at $u^{\prime}$ and the second part $\beta_{2}$ which starts at $u^{\prime}$ and ends at $u$ (see Fig. 10b).

Let $\left(s_{y}^{\prime}\right)_{1}$ (resp. $\left.\left(s_{y}^{\prime}\right)_{2}\right)$ be the skeleton after the action of the braids induced by $\beta_{1}$ (resp. $\beta_{y}$ ) in the arrangement $\mathcal{L}^{\prime}$ and let $\left(s_{y}\right)_{1}$ (resp. $\left.\left(s_{y}\right)_{2}\right)$ be this skeleton after the action of the braids induced by $\beta_{1}$ (resp. $\beta_{y}$ ) in the arrangement $\mathcal{L}$.

Note that the fibers over $x(y)-\varepsilon$ in $\mathcal{L}$ and in $\mathcal{L}^{\prime}$ differ only by one point - in $\mathcal{L}$ there is an additional point in the fiber, being the lowest point (numerated by 1 ) and 
representing the line $L$. Since the braids induced by $\beta_{1}$ in the arrangement $\mathcal{L}$ do not induce any motion on this point in the fiber, numerated by 1 (as the line $L$ does not intersect the other components in the section between $x(y)$ and $\left.u^{\prime}\right)$, the skeletons $\left(s_{y}^{\prime}\right)_{1}$ and $\left(s_{y}\right)_{1}$ are the same, except for that all the indices in $\left(s_{y}\right)_{1}$ are increased by 1.

Note that the braid induced by the path $\beta_{2}$ in $\mathcal{L}^{\prime}$ is

$$
\Delta^{-1}\langle v+1, v+m-1\rangle
$$

as $p \in \mathcal{L}^{\prime}$ is an intersection point with multiplicity $m-1$. On the other hand, the braid induced by the path $\beta_{2}$ in $\mathcal{L}$ is:

$$
\begin{aligned}
B_{2} \doteq & \Delta^{-1}\langle 1,2\rangle \cdots \Delta^{-1}\langle v, v+1\rangle \Delta^{-1}\langle v+1, v+m\rangle \\
& \cdot \Delta^{-1}\langle v+m, v+m+1\rangle \cdots \Delta^{-1}\langle n-1, n\rangle
\end{aligned}
$$

Now, when performing $B_{2}$ on $\left(s_{y}\right)_{1}$, recall that $\beta_{2}$ is a path beneath all the intersection points on $L$ (that is, there are no intersection points on $L$ outside the section $\left.\left\{a \in \mathbb{C}^{2}: u^{\prime}<x(a)<u\right\}\right)$. Since $L$ is almost vertical with a negative slope, the braid $B_{2}$ induces the motion $\delta_{n}^{-1}$ on the point numerated by 1 in the arrangement $\mathcal{L}$ (in the fiber over $u^{\prime}$ ), that is, the point 1 performs a $180^{\circ}$ rotation clockwise, eventually turning into the point numerated by $n$. Moreover, as the point 1 (in the fiber over $\left.u^{\prime}\right)$ is not involved in the skeleton $\left(s_{y}\right)_{1}$, the only braid that does affect $\left(s_{y}\right)_{1}$ is $\Delta^{-1}\langle v+1, v+m\rangle$. At the stage of applying $\Delta^{-1}\langle v+1, v+m\rangle($ in $\mathcal{L})$, the point numbered as 1 is then numbered as $v+1$ and the skeleton $\left(s_{y}\right)_{1}$ is passing beneath it. The braid $\Delta^{-1}\langle v+1, v+m\rangle$ sends the point $v+1$ to the point $v+m$ and the effect on the skeleton itself is as the effect of $\Delta^{-1}\langle v+1, v+m-1\rangle$. This means that the skeletons $\left(s_{y}^{\prime}\right)_{2}$ and $\left(s_{y}\right)_{2}$ are the same, but in the model of $\left(s_{y}\right)_{2}$ there will be one additional point corresponding to $L$, which will be the highest point.

Part (II): In this part we prove that $\pi_{1}\left(\mathbb{C}^{2}-\mathcal{L}, u\right)$ has a conjugation-free geometric presentation, using the fact that $\pi_{1}\left(\mathbb{C}^{2}-\mathcal{L}^{\prime}, u\right)$ has a conjugation-free presentation. Note that this is the same method used in the second part of [11, Proposition 2.2], and we bring it here for the convenience of the reader.

We know that $\pi_{1}\left(\mathbb{C}^{2}-\mathcal{L}^{\prime}, u\right)$ has a conjugation-free geometric presentation; hence we have that by a simplification process starting from the corresponding Zariski-van Kampen presentation, one can reach a presentation without conjugations. If we imitate the simplification process of the presentation of $\pi_{1}\left(\mathbb{C}^{2}-\mathcal{L}^{\prime}, u\right)$ for the presentation of $\pi_{1}\left(\mathbb{C}^{2}-\mathcal{L}, u\right)$, the cases in which we need to use the relations induced by the point $p$ are the relations that have been simplified by using the relations induced by $p$ before adding the line $L$. Recall that the Lefschetz pair of $p$ in $\mathcal{L}$ is $[v+1, v+m]$, and in $\mathcal{L}^{\prime}$ the corresponding Lefschetz pair is $[v+1, v+m-1]$. Denote by $\left\{\Gamma_{i}\right\}$ the geometric generators both in $\pi_{1}\left(\mathbb{C}^{2}-\mathcal{L}^{\prime}, u\right)$ and $\pi_{1}\left(\mathbb{C}^{2}-\mathcal{L}, u\right)$.

Thus, in $\pi_{1}\left(\mathbb{C}^{2}-\mathcal{L}^{\prime}, u\right)$, the relations induced by $p$ are:

$$
R_{p}:\left[\Gamma_{v+1}, \Gamma_{v+2}, \ldots, \Gamma_{v+m-1}\right]=e
$$


Since in the fiber over $u$ in the arrangement $\mathcal{L}$, the line $L$ is numbered as $n$ (and the numeration of the other lines was not changed), the relations induced by $p$ in $\pi_{1}\left(\mathbb{C}^{2}-\mathcal{L}, u\right)$ are:

$$
\tilde{R}_{p}:\left[\Gamma_{v+1}, \Gamma_{v+2}, \ldots, \Gamma_{v+m-1}, \Gamma_{n}\right]=e
$$

We can divide the relations in $\pi_{1}\left(\mathbb{C}^{2}-\mathcal{L}^{\prime}, u\right)$ into two subsets:

(1) Relations that during the simplification process contain the subword

$$
\Gamma_{v+1}^{-1} \cdots \Gamma_{v+m-2}^{-1} \Gamma_{v+m-1} \Gamma_{v+m-2} \cdots \Gamma_{v+1}
$$

(2) Relations that do not contain the above subword during its simplification process.

For the second subset, the simplification process will be identical before adding the line $L$ and after it, since all the other relations induced by points of $\mathcal{L}^{\prime}$ have not been changed by adding the line $L$.

For the first subset, let $R$ be a relation from this subset. Except for applying the relations induced by $p$, the rest of the simplification process is identical to the one before adding the line. The only change is in the step of applying $R_{p}$. In this step, before adding the line $L$, the generator $\Gamma_{n}$ has not been involved in $R_{p}$, but after adding the line $L$, it appears in $\tilde{R}_{p}$. Hence, for applying $\tilde{R}_{p}$, we have to conjugate the relation $R$ by $\Gamma_{n}$, and using the commutative relations which $\Gamma_{n}$ is involved, we can diffuse $\Gamma_{n}$ into the relation $R$, so we can use the relation $\tilde{R}_{p}$ instead of $R_{p}$.

Hence, we can simplify all the conjugations in all the relations in $\pi_{1}\left(\mathbb{C}^{2}-\mathcal{L}, u\right)$ as we simplify the relations in $\pi_{1}\left(\mathbb{C}^{2}-\mathcal{L}^{\prime}, u\right)$, so we get a conjugation-free geometric presentation, as needed.

Step 3.9 For $u=x\left(p_{0}\right)+\varepsilon, \pi_{1}\left(\mathbb{C}^{2}-\mathcal{L}, u\right)$ has a conjugation-free geometric presentation.

Proof The proof of this step is almost identical to the proof of Step 3.8. Again, all the relations induced by the intersection points on $L$ have no conjugations. As for the intersection points to the right of $u$, since the intersection points on $L$ are not affecting any of these intersection points, we can consider the resulting skeletons as skeletons of $\mathcal{L}^{\prime}$ except for that now all the indices are increased by 1 (as the line $L$ is numbered as 1 ). We now have to treat the relations induced by the intersection points which are to the left of $L$.

However, the treatment is almost identical to the treatment of Step 3.8: for an intersection point $y$, which is to the left of $p^{\prime}$, we consider the two initial skeletons in the fiber over $x(y)+\varepsilon$ in $\mathcal{L}^{\prime}$ and in $\mathcal{L}$, which are the same, except for that in $\mathcal{L}$ there is one additional point corresponding to $L$, which will be the highest point. Now, we consider a path $\beta_{y}$ from $x(y)+\varepsilon$ to $u$ and we divide it into two parts: the first part $\beta_{1}$, from $x(y)+\varepsilon$ to $x\left(p^{\prime}\right)-\varepsilon$, and the second part $\beta_{2}$, from $x\left(p^{\prime}\right)-\varepsilon$ to $u$. As before, the first part does not induce any difference between the two above skeletons. The second part $\beta_{2}$ causes eventually that the highest point in the fiber in $\mathcal{L}$ performs a $180^{\circ}$ rotation counterclockwise, while as for the rest of the skeleton, the composition 
of the braids corresponding to this part in $\mathcal{L}$ induced the same operation as if it were in $\mathcal{L}^{\prime}$. This means that the final skeletons induced by $y$ (the one in $\mathcal{L}^{\prime}$ and the second in $\mathcal{L}$ ) are the same, except for that all the indices in the second are increased by 1 . Now we can use the same simplification process with the method described in Part (II) of the proof of Step 3.8.

Recall that $N$ denotes the set of the images of the singular points (with respect to the projection $\pi$ ) on $\ell$. We now prove that $\pi_{1}\left(\mathbb{C}^{2}-\mathcal{L}, u\right)$ has a conjugation-free geometric presentation for every basepoint $u$ that belongs to one of the following domains:

- $D_{1}=\left\{u \in \ell-N: x\left(p^{\prime}\right)<u<x\left(p_{0}\right)\right\}$.

- $D_{2}=\left\{u \in \ell-N: x\left(p_{0}\right)<u\right\}$.

- $D_{3}=\left\{u \in \ell-N: x\left(p^{\prime}\right)>u\right\}$.

Step 3.10 For $u \in D_{1}, \pi_{1}\left(\mathbb{C}^{2}-\mathcal{L}, u\right)$ has a conjugation-free geometric presentation.

Proof If $u \in D_{1}$, we can use Remark 3.5(II), stating that when we pass $u$ "below" the image of a node (with respect to the projection), then the conjugation-free property is preserved. Then, $\pi_{1}\left(\mathbb{C}^{2}-\mathcal{L}, u\right)$ is conjugation-free, since we can either start from the basepoint $x\left(p^{\prime}\right)-\varepsilon$ (as we know by Step 3.8 that $\pi_{1}\left(\mathbb{C}^{2}-\mathcal{L}, x\left(p^{\prime}\right)-\varepsilon\right.$ ) is conjugation-free) and "pass" $u$ to the right, going only below nodes till we almost reach $x(p)$; or we can start from the basepoint $x\left(p_{0}\right)+\varepsilon$ (as we know by Step 3.9 that $\pi_{1}\left(\mathbb{C}^{2}-\mathcal{L}, x\left(p_{0}\right)+\varepsilon\right)$ is conjugation-free) and "pass" $u$ to the left, going only below nodes till we almost reach $x(p)$ (from its other side).

Step 3.11 For $u \in D_{2}, \pi_{1}\left(\mathbb{C}^{2}-\mathcal{L}, u\right)$ has a conjugation-free geometric presentation.

Proof The proof of this step is based on proving the following three claims:

(1) All the skeletons induced by the intersection points not on $L$ are the same in $\mathcal{L}$, as if they were computed in $\mathcal{L}^{\prime}$, except for that all the corresponding indices in $\mathcal{L}$ are increased by 1 .

(2) All the relations induced by the nodes on $L$ have no conjugations.

(3) The relation induced by $p \in L$ has no conjugations.

If we prove these claims, then we are done, as we can use the same simplification process done for the skeletons associated to the intersection points not on $L$ in $\pi_{1}\left(\mathbb{C}^{2}-\mathcal{L}^{\prime}, u\right)$, as we have done in Part (II) in the proof of Step 3.8.

As for claim (1), the proof is essentially the same as in Step 3.9. We now prove claim (2) by induction on the location of the basepoint $u$ with respect to the intersection points of $\mathcal{L}$ to the right of $p_{0}$. Let us numerate the intersection points of $\mathcal{L}$ in the domain $\left\{m \in \mathbb{C}^{2}: x(m) \geq x\left(p_{0}\right)\right\}$ by $q_{0}=p_{0}, q_{1}, \ldots, q_{k}$, where $x\left(q_{i}\right)<x\left(q_{j}\right)$ for $i<j$. Now, as was proved in Step 3.9, the claim is correct when $u \in\left\{m \in \ell: x\left(q_{0}\right)<\right.$ $\left.x(m)<x\left(q_{1}\right)\right\}$ which is the base of the induction. Assume that the claim is correct for all $u \in\left\{m \in \ell: x\left(q_{0}\right)<x(m)<x\left(q_{i}\right)\right\}$. We now prove that the claim is correct when $u$ is in the next domain $\left\{m \in \ell: x\left(q_{i}\right)<x(m)<x\left(q_{i+1}\right)\right\}$. 
Assume that $u=x\left(q_{i}\right)-\varepsilon$, and that in the fiber over $u$, the local numeration of the lines intersecting in $q_{i}$ is $a, a+1, \ldots, a+s=b$, where $1<a$ (as the line $L$ is always numbered as 1 in the domain $\left.\left\{m \in \ell: x(m)>x\left(p_{0}\right)\right\}\right)$. By Remark 3.5(I), when we move $u$ to $u^{\prime}=x\left(q_{i}\right)+\varepsilon$, the induced isomorphism affects only the geometric generators $\Gamma_{a}, \ldots, \Gamma_{b}$ by a Hurwitz move. Explicitly, the isomorphism is:

$$
f_{3}^{\prime}\left(\Gamma_{i}\right)=\left\{\begin{array}{cl}
\Gamma_{i} & i<a \text { or } i>b \\
\Gamma_{b}^{\prime} \cdots \Gamma_{a+1}^{\prime} \Gamma_{a}^{\prime} \Gamma_{a+1}^{\prime-1} \cdots \Gamma_{b}^{\prime-1} & i=a+s=b \\
\Gamma_{b}^{\prime} \cdots \Gamma_{a+2}^{\prime} \Gamma_{a+1}^{\prime} \Gamma_{a+2}^{\prime-1} \cdots \Gamma_{b}^{\prime-1} & i=b-1 \\
\vdots & \vdots \\
\Gamma_{b}^{\prime} \Gamma_{b-1}^{\prime} \Gamma_{b}^{\prime-1} & i=a+1 \\
\Gamma_{b}^{\prime} & i=a,
\end{array}\right.
$$

where $\left\{\Gamma_{i}^{\prime}\right\}$ are the geometric generators of $\pi_{1}\left(\mathbb{C}^{2}-\mathcal{L}, u^{\prime}\right)$. Note that the isomorphism $f_{3}$ described in Eq. (5) is associated to moving a basepoint from right to left, while $f_{3}^{\prime}$ is associated to moving a basepoint from left to right; explicitly, $f_{3}^{\prime}=f_{3}^{-1}$.

We split the proof into two cases.

Case (1): There is no line passing through $p$ and $q_{i}$.

When the basepoint is $u$, then the relations induced by the nodes on $L$ are:

$$
\left[\Gamma_{1}, \Gamma_{j}\right]=e, \quad a \leq j \leq b
$$

(by the conjugation-free property). Thus, the above isomorphism $f_{3}^{\prime}$ affects these commutative relations in the following way:

$$
\begin{gathered}
{\left[\Gamma_{1}, \Gamma_{a}\right]=e \rightarrow\left[\Gamma_{1}^{\prime}, \Gamma_{b}^{\prime}\right]=e,} \\
{\left[\Gamma_{1}, \Gamma_{a+1}\right]=e \rightarrow\left[\Gamma_{1}^{\prime}, \Gamma_{b}^{\prime} \Gamma_{b-1}^{\prime} \Gamma_{b}^{\prime-1}\right] \stackrel{\left[\Gamma_{1}^{\prime}, \Gamma_{b}^{\prime}\right]=e}{=}\left[\Gamma_{1}^{\prime}, \Gamma_{b-1}^{\prime}\right]=e,} \\
{\left[\Gamma_{1}, \Gamma_{a+2}\right]=e \rightarrow\left[\Gamma_{1}^{\prime}, \Gamma_{b}^{\prime} \Gamma_{b-1}^{\prime} \Gamma_{b-2}^{\prime} \Gamma_{b-1}^{\prime-1} \Gamma_{b}^{\prime-1}\right] \stackrel{\left[\Gamma_{1}^{\prime}, \Gamma_{b}^{\prime}\right]=\left[\Gamma_{1}^{\prime}, \Gamma_{b-1}^{\prime}\right]=e}{=}\left[\Gamma_{1}^{\prime}, \Gamma_{b-2}^{\prime}\right]=e,}
\end{gathered}
$$

and we continue in the same way for the other relations in order to get rid of the conjugations.

If there are nodes on $L$ such that the lines passing through them are numbered as 1 and $m$ (when numerated locally over $u^{\prime}$ ), when $m<a$ or $m>b$, then the relation $\left[\Gamma_{1}, \Gamma_{m}\right]=e$ in $\pi_{1}\left(\mathbb{C}^{2}-\mathcal{L}, u\right)$ is left unchanged in $\pi_{1}\left(\mathbb{C}^{2}-\mathcal{L}, u^{\prime}\right)$, i.e. the corresponding relation still has no conjugations.

Case (2): There is a line passing through $p$ and $q_{i}$.

Assume that the line passing through $p$ and $q_{i}$ is numbered, locally over $u$, as $a+k$. Therefore, when the basepoint is $u$, the relations induced by the nodes on $L$ are:

$$
\left[\Gamma_{1}, \Gamma_{x}\right]=e, \quad a \leq x \leq b, x \neq a+k
$$

After the isomorphism, we know in any case that the relations: 


$$
\left[\Gamma_{a}^{\prime}, \Gamma_{a+1}^{\prime}, \Gamma_{a+2}^{\prime}, \ldots, \Gamma_{b}^{\prime}\right]=e
$$

exist (these are the relations induced by $q_{i}$ ). The treatment in the relations

$$
\left[\Gamma_{1}, \Gamma_{x}\right]=e, \quad a \leq x \leq a+k-1
$$

is exactly as in case (1). We now treat the remaining relations, i.e. the relations

$$
\left[\Gamma_{1}, \Gamma_{x}\right]=e, \quad a+k+1 \leq x \leq b .
$$

Now,

$$
\begin{gathered}
{\left[\Gamma_{1}, \Gamma_{b}\right]=e \rightarrow\left[\Gamma_{1}^{\prime}, \Gamma_{b}^{\prime} \Gamma_{b-1}^{\prime} \cdots \Gamma_{a+1}^{\prime} \Gamma_{a}^{\prime} \Gamma_{a+1}^{\prime-1} \cdots \Gamma_{b-1}^{\prime-1} \Gamma_{b}^{\prime-1}\right] \stackrel{\text { Eqn. }}{=}\left[\Gamma_{1}^{\prime}, \Gamma_{a}^{\prime}\right]=e .} \\
{\left[\Gamma_{1}, \Gamma_{b-1}\right]=e \rightarrow\left[\Gamma_{1}^{\prime}, \Gamma_{b}^{\prime} \Gamma_{b-1}^{\prime} \cdots \Gamma_{a+2}^{\prime} \Gamma_{a+1}^{\prime} \Gamma_{a+2}^{-1} \cdots \Gamma_{b-1}^{\prime-1} \Gamma_{b}^{\prime-1}\right]} \\
=\left[\Gamma_{a}^{\prime} \Gamma_{1}^{\prime} \Gamma_{a}^{\prime-1}, \Gamma_{a}^{\prime} \Gamma_{b}^{\prime} \Gamma_{b-1}^{\prime} \cdots \Gamma_{a+2}^{\prime} \Gamma_{a+1}^{\prime} \Gamma_{a+2}^{\prime-1} \cdots \Gamma_{b-1}^{-1} \Gamma_{b}^{\prime-1} \Gamma_{a}^{\prime-1}\right] \\
\quad \stackrel{\text { Eqns. (7),(8) }}{=}\left[\Gamma_{1}^{\prime}, \Gamma_{a+1}^{\prime}\right]=e \\
{\left[\Gamma_{1}, \Gamma_{b-2}\right]=e \rightarrow\left[\Gamma_{1}^{\prime}, \Gamma_{b}^{\prime} \Gamma_{b-1}^{\prime} \cdots \Gamma_{a+3}^{\prime} \Gamma_{a+2}^{\prime} \Gamma_{a+3}^{-1} \cdots \Gamma_{b-1}^{\prime-1} \Gamma_{b}^{\prime-1}\right]} \\
=\left[\Gamma_{a}^{\prime} \Gamma_{a+1}^{\prime} \Gamma_{1}^{\prime} \Gamma_{a+1}^{-1} \Gamma_{a}^{\prime-1}, \Gamma_{a}^{\prime} \Gamma_{a+1}^{\prime} \Gamma_{b}^{\prime} \Gamma_{b-1}^{\prime} \cdots \Gamma_{a+3}^{\prime} \Gamma_{a+2}^{\prime} \Gamma_{a+3}^{-1} \cdots\right. \\
\left.\quad \cdots \Gamma_{b-1}^{-1} \Gamma_{b}^{\prime-1} \Gamma_{a+1}^{\prime-1} \Gamma_{a}^{\prime-1}\right] \stackrel{\text { Eqns. (7) }}{=} \stackrel{-(9)}{=}\left[\Gamma_{1}^{\prime}, \Gamma_{a+2}^{\prime}\right]=e
\end{gathered}
$$

and we continue in the same way for the relations induced by the other nodes.

As in the previous case, if there are nodes on $L$ such that the lines passing through them are (when numerated locally over $u$ ) 1 and $m$, when $m<a$ or $m>b$, then the relation $\left[\Gamma_{1}, \Gamma_{m}\right]=e$ in $\pi_{1}\left(\mathbb{C}^{2}-\mathcal{L}, u\right)$ is left unchanged in $\pi_{1}\left(\mathbb{C}^{2}-\mathcal{L}, u^{\prime}\right)$, i.e. the corresponding relation still has no conjugations.

We now prove claim (3), that the relations induced by $p \in L$ have no conjugations. Recall that the point $p$ is an intersection point of multiplicity $m>2$. The proof is similar to the proof of claim (2), i.e., it is by induction on the location of the basepoint $u$ with respect to the intersection points of $\mathcal{L}$ to the right of $p_{0}$. Again, we know that this claim holds when $u \in\left\{m \in \ell: x\left(q_{0}\right)<x(m)<x\left(q_{1}\right)\right\}$, by Step 3.9, which is the base of the induction. Assume that the claim is correct for all $u \in\left\{m \in \ell: x\left(q_{0}\right)<x(m)<x\left(q_{i}\right)\right\}$. We now prove that it is correct when $u$ is in the domain $\left\{m \in \ell: x\left(q_{i}\right)<x(m)<x\left(q_{i+1}\right)\right\}$.

Assume that $u=x\left(q_{i}\right)-\varepsilon$, and denote $u^{\prime}=x\left(q_{i}\right)+\varepsilon$. Let $\beta_{u}$ be a path starting at $x(p)+\varepsilon$ and ending at $u$ (see Fig. 11). Denote by $B_{u}$ the braid that is induced by the path $\beta_{u}$ in $\mathcal{L}$ (i.e. the Lefschetz diffeomorphism $\psi_{\beta_{u}}$; see Definition 2.10).

Assume that in the fiber over $u$, the local numeration of the lines intersecting in $q_{i}$ is $a, a+1, \ldots, a+s=b$, where $1<a$ (as the line $L$ is always numbered as 1 in the domain $\left.\left\{m \in \ell: x(m)>x\left(p_{0}\right)\right\}\right)$. Recall that the Lefschetz pair of the point $p$ in $\mathcal{L}$ over $x(p)+\varepsilon$ is $[v+1, v+m]$. 


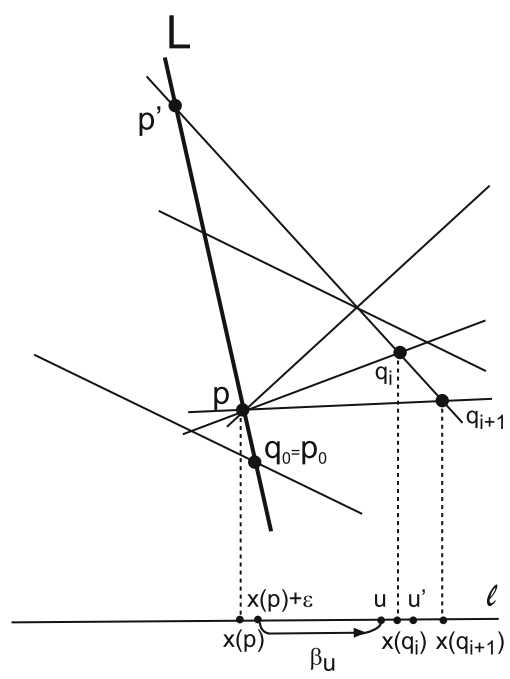

Fig. 11 Notations for the proof of claim (3) of Step 3.11

When the basepoint is $u$, the simplified relation induced by the point $p$ is:

$$
\left[\Gamma_{1}, \Gamma_{B_{u}(v+2)}, \Gamma_{B_{u}(v+3)}, \ldots, \Gamma_{B_{u}}(v+m)\right]=e,
$$

by the conjugation-free property of $\pi_{1}\left(\mathbb{C}^{2}-\mathcal{L}, u\right)$, which is the induction hypothesis (the notation $B_{u}(x)$ refers to the numeration of the point $x$ after applying the braid $B_{u}$ on it, when considering the braid group as modeled by the group of diffeomorphisms on a punctured disk, where $x$ is one of the punctures; see Definition 2.8). Note that when passing to the basepoint $u^{\prime}$, the induced isomorphism is $f_{3}^{\prime}$ as described earlier [see Eq. (6)]. However, note that $f_{3}^{\prime}$ affects at most one of the generators $\Gamma_{B_{u}(x)}$, where $v+2 \leq x \leq v+m$, since there is at most one line passing through both $p$ and $q_{i}$. We now divide the proof into two cases: when there is no line passing through $p$ and $q_{i}$ and when there is one.

Case (1): If there is no line passing through $p$ and $q_{i}$, then the isomorphism $f_{3}^{\prime}$ does not affect relation (10), i.e. all the generators $\Gamma_{i}$ that participate in the relation are sent to $\Gamma_{i}^{\prime}$. This means that the relations induced by $p$ have no conjugations in $\pi_{1}\left(\mathbb{C}^{2}-\mathcal{L}, u^{\prime}\right)$.

Case (2): There is a line passing through $p$ and $q_{i}$.

Assume that this line is numbered over $u$ as $B_{u}(j)=a+t$ (where $0 \leq t \leq s$, $v+2 \leq j \leq v+m$ ), that is, only the generator $\Gamma_{a+t}$ is affected by $f_{3}^{\prime}$ and the other generators $\Gamma_{B_{u}(x)}, v+2 \leq x \leq v+m, x \neq j$, are sent to $\Gamma_{B_{u}(x)}^{\prime}$. Now,

$$
f_{3}^{\prime}\left(\Gamma_{B_{u}(j)}\right)=f_{3}^{\prime}\left(\Gamma_{a+t}\right) \doteq \Gamma_{b}^{\prime} \cdots \Gamma_{b-t+1}^{\prime} \Gamma_{b-t}^{\prime} \Gamma_{b-t+1}^{\prime-1} \cdots \Gamma_{b}^{\prime-1}
$$

Note that over $u^{\prime}$, the line that passes through $p$ and $q_{i}$, is numbered as $b-t$. This means that the lines numbered as $b, b-1, \ldots, b-t+1$ over $u^{\prime}$, do not pass 
through $p$ and therefore they intersect the line $L$ in nodes. By claim (2), we have in $\pi_{1}\left(\mathbb{C}^{2}-\mathcal{L}, u^{\prime}\right)$ that $\left[\Gamma_{1}^{\prime}, \Gamma_{x}^{\prime}\right]=e, b-t+1 \leq x \leq b$.

Thus, the relation induced by $p$ in $\pi_{1}\left(\mathbb{C}^{2}-\mathcal{L}, \overline{u^{\prime}}\right)$ is now:

$$
\breve{\mathcal{R}}_{p}:\left[\Gamma_{1}^{\prime}, \Gamma_{B_{u}(v+2)}^{\prime}, \ldots, \Gamma_{B_{u}(j-1)}^{\prime}, f_{3}^{\prime}\left(\Gamma_{B_{u}(j)}\right), \Gamma_{B_{u}(j+1)}^{\prime}, \ldots, \Gamma_{B_{u}(v+m)}^{\prime}\right]=e .
$$

We claim that we can perform the same simplification process, done in $\pi_{1}\left(\mathbb{C}^{2}-\mathcal{L}^{\prime}, u^{\prime}\right)$, for the relation $\mathcal{R}_{p}$ induced by $p$, after moving from $u$ to $u^{\prime}$. Indeed, the relation $\mathcal{R}_{p}$ is

$$
\mathcal{R}_{p}:\left[\Gamma_{B_{u}(v+1)}^{\prime}, \ldots, \Gamma_{B_{u}(j-2)}^{\prime}, f_{3}^{\prime}\left(\Gamma_{B_{u}(j-1)}\right), \Gamma_{B_{u}(j)}^{\prime}, \ldots \Gamma_{B_{u}(v+m-1)}^{\prime}\right]=e
$$

(recall that we have to increase the indices by 1 when passing from $\mathcal{L}^{\prime}$ to $\mathcal{L}$ when the basepoint is to the right of $\left.x\left(p_{0}\right)\right)$.

We have to consider two cases: the first is that during the simplification process of $\mathcal{R}_{p}$, we are simplifying $f_{3}^{\prime}\left(\Gamma_{B_{u}(j-1)}\right)$; and the second is that during the simplification process of $\mathcal{R}_{p}$, we are simplifying a product of $f_{3}^{\prime}\left(\Gamma_{B_{u}(j-1)}\right) \cdot \Gamma_{B_{u}(j-2)}^{\prime}$, which might be turned, in $\breve{\mathcal{R}}_{p}$, to a product of $f_{3}^{\prime}\left(\Gamma_{B_{u}(j)}\right) \cdot \Gamma_{1}^{\prime} \cdot \Gamma_{B_{u}(j-1)}^{\prime}$. Now, only the second case can cause problems (as the first case does not involve $\Gamma_{1}^{\prime}$ ). However, note that in the product $f_{3}^{\prime}\left(\Gamma_{B_{u}(j)}\right) \cdot \Gamma_{1}^{\prime}$, we can diffuse $\Gamma_{1}^{\prime}$ till it reaches the generator $\Gamma_{b-t}^{\prime}$ (since $\left[\Gamma_{1}^{\prime}, \Gamma_{x}^{\prime}\right]=e$, for all $b-t+1 \leq x \leq b$ as was explained above) and now apply the same simplification process as was done in $\pi_{1}\left(\mathbb{C}^{2}-\mathcal{L}^{\prime}, u^{\prime}\right)$.

Therefore, the relation induced by $p$ has no conjugations and now we can use the method described in Part (II) in the proof of Step 3.8.

Step 3.12 For $u \in D_{3}, \pi_{1}\left(\mathbb{C}^{2}-\mathcal{L}, u\right)$ has a conjugation-free geometric presentation.

Proof The proof of this step is similar to the proof of Step 3.11. The only difference is that now we use the isomorphism $f_{3}$ (and not $f_{3}^{\prime}$ ), as we move (during the inductive step of the proofs of claims (2) and (3)) from the basepoint $x\left(q_{i}\right)+\varepsilon$ to the basepoint $x\left(q_{i}\right)-\varepsilon$. However, as can be easily seen, we can use the same techniques for proving these claims.

The above series of steps completes the proof of Proposition 2.18(1).

\section{Preservation of conjugation-freeness: the case of real $\mathrm{CL}$ arrangements}

The goal of this section is to prove Proposition 2.18(2): i.e., under certain conditions, if we add a line $L$ to a $\mathrm{CL}$ arrangement $\mathcal{A}$ whose fundamental group is conjugationfree, such that $L$ passes through at most one singular point of $\mathcal{A}$, then the fundamental group of $\mathcal{A} \cup L$ is also conjugation-free. The additional condition, that is not required in the case of real line arrangements, is that $\beta(\mathcal{A} \cup L)=0$. However, we will see in Sect. 5.2 that this condition can be weakened. Moreover, in the course of the proof, we prove other important results for CL arrangements, such as the commutation of the 
generator associated to the conic with all the other geometric generators (associated to the lines) in $\pi_{1}\left(\mathbb{C}^{2}-\mathcal{A}\right)$.

When trying to follow the proof given in the previous section (for real line arrangements), in addition to the operations $h_{1}, h_{2}, h_{3}$, which can also be operated on a real CL arrangement, we have to consider the following new operation:

- $h_{4}$ : If a real rotation of a line causes a tangency point between this line and the conic, perform a rotation in $\mathbb{C}^{2}$ over the possible tangency point;

Moreover, we have to reconsider the following operations:

- $h_{1}$ : Rotating a line over a multiple point, as in the case of real line arrangements.

- $h_{3}$ : Changing the basepoint $u \in \ell-N$, now when moving $u$ from one side of a branch point to its other side.

We note that the proof of the preservation of the conjugation-free property of the operation $h_{2}$ (rotating a line over another line) holds intact also for the CL arrangements specified in Proposition 2.18(2), as it involves only the generators of the lines. The proof of the preservation of the conjugation-free property of the operation $h_{3}$ will have to be changed, as we will now deal with complex points too.

Lemma 4.1 For a real CL arrangement $\mathcal{A}$ with one conic, the operation $h_{1}$ preserves the conjugation-free property.

Proof Let $b_{1}, b_{2}$ be the two branch points of the conic with respect to the projection. We have to consider two cases: the first is that during the rotation of the line $L \in \mathcal{A}$, $L$ does not pass through one of the $b_{i}$ 's. The second is that it does pass through one of them.

As for the first case, the proof is the same as the proof of Lemma 3.3. As for the second case, we have the situation depicted in Fig. 12a.

As before, the isomorphism $\pi_{1}\left(\mathbb{C}^{2}-\mathcal{A}, u\right) \rightarrow \pi_{1}\left(\mathbb{C}^{2}-h_{1}(\mathcal{A}), u\right)$ is $\Gamma_{i} \mapsto \Gamma_{i}^{\prime}$. Thus, the only relation that might be changed by this rotation is the one induced by the branch point $b$ : after considering the complex points of the fiber as real points, the relation might be transposed to the following: $\left\{\Gamma_{i}=\Gamma_{i+1}\right\} \mapsto\left\{\Gamma_{i-1}^{\prime} \Gamma_{i}^{\prime} \Gamma_{i-1}^{\prime-1}=\Gamma_{i+1}^{\prime}\right\}$, where $\Gamma_{i-1}^{\prime}$ is the generator of the line and $\Gamma_{i}^{\prime}, \Gamma_{i+1}^{\prime}$ are the generators of the conic in $\pi_{1}\left(\mathbb{C}^{2}-h_{1}(\mathcal{A}), u\right)$ (see Fig. 12b, c). However, due to the isomorphism above, we still have the relation $\left[\Gamma_{i-1}^{\prime}, \Gamma_{i}^{\prime}\right]=e\left(\right.$ or $\left[\Gamma_{i-1}^{\prime}, \Gamma_{i+1}^{\prime}\right]=e$; note that $\pi_{1}\left(\mathbb{C}^{2}-\mathcal{A}\right)$ is conjugation-free) and thus the resulting relation induced by the branch point has no conjugations. So, we get that the presentation of $\pi_{1}\left(\mathbb{C}^{2}-h_{1}(\mathcal{A})\right)$ is also conjugationfree.

Lemma 4.2 For a real CL arrangement $\mathcal{A}$ with one conic, the operation $h_{4}$ preserves the conjugation-free property.

Proof Given a line $L \in \mathcal{A}$ which we have to rotate, the rotation of $L$ in $\mathbb{R}^{2}$ might not be an equisingular deformation, as at a particular point during the rotation, $L$ may be tangent to the conic. In this case, we change the rotation in $\mathbb{R}^{2}$ to the following equisingular deformation, according to the local model appearing in Fig. 13a. 

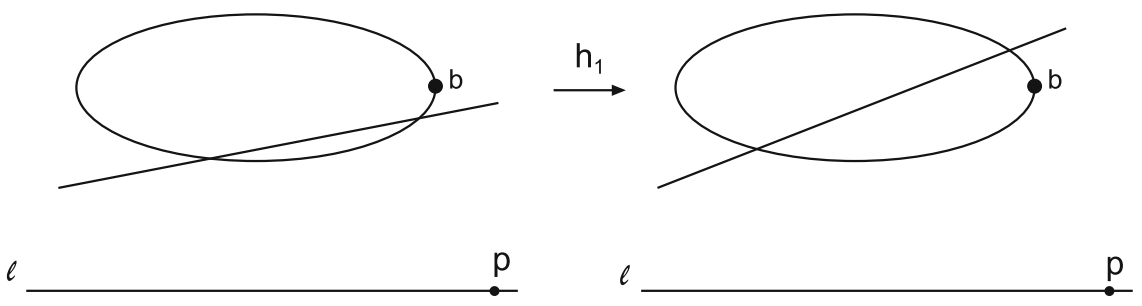

(a)

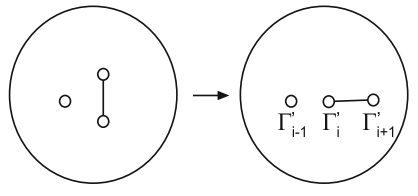

(b)

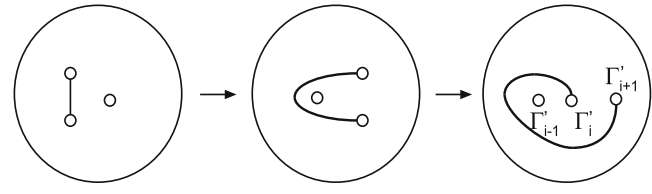

(c)

Fig. 12 Part $\mathbf{a}$ is a rotation of a line over a branch point $b$ and Part $\mathbf{b}$ and $\mathbf{c}$ are its effects on the skeleton associated to the branch point $b$

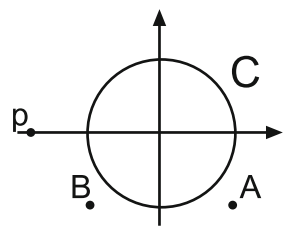

(a)

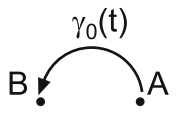

(b)

Fig. 13 Part a is a local model for a complex rotation: $C=\left\{(x, y) \in \mathbb{C}^{2}: x^{2}+y^{2}=1\right\}$, $p=(-2,0)$. Part b illustrates the path $\gamma_{0}(t)$ used to rotate the line

Let $C=\left\{(x, y) \in \mathbb{C}^{2}: x^{2}+y^{2}=1\right\}, p=(-2,0), A=(1,-1)$ and $B=(-1,-1)$. Let $\gamma_{0}:[0,1] \rightarrow \mathbb{C}^{2}$ be half a circle of radius 1 on the plane $\{y=-1\}$ in $\mathbb{C}^{2}$ which starts at $A$ and ends at $B$ (see Fig. 13b). Let $L(t)$ be the family of lines passing through $p$ and $\gamma_{0}(t)$, for $0 \leq t \leq 1$. Obviously, $C \cup L(t)$ is an equisingular deformation and thus $\pi_{1}\left(\mathbb{C}^{2}-(C \cup L(0))\right) \cong \pi_{1}\left(\mathbb{C}^{2}-(C \cup L(1))\right)$. Denote by $h_{4}$ this operation after which $L=L(1)$.

We have to prove that if $\pi_{1}\left(\mathbb{C}^{2}-\mathcal{A}, u\right)$ is conjugation-free, then also $\pi_{1}\left(\mathbb{C}^{2}-h_{4}(\mathcal{A}), u\right)$ is conjugation-free. We have to check, for all the initial skeletons associated to the singular points to the right of $p_{1}, p_{2}$, that the braids that are applied on them, while passing below $x\left(p_{1}\right), x\left(p_{2}\right)$, along a path $\beta$, are the same in the CL arrangements $\mathcal{A}$ and $h_{4}(\mathcal{A})$; see the models depicted in Fig. 14a, b.

Before the rotation of $L$ (see Fig. 14a), the braids that are applied on the skeletons while passing below $x\left(p_{1}\right)$ and $x\left(p_{2}\right)$, along a path $\beta$, are $\Delta\langle t, t+1\rangle \cdot \Delta\langle t, t+1\rangle=(H(\sigma))^{2}$, where $t$ is the local index of the point in the fiber which corresponds to the line $L, t+1$ is the local index of the "lower" generator of the conic and $\sigma$ is the segment connecting these two points in the fiber. In order to 

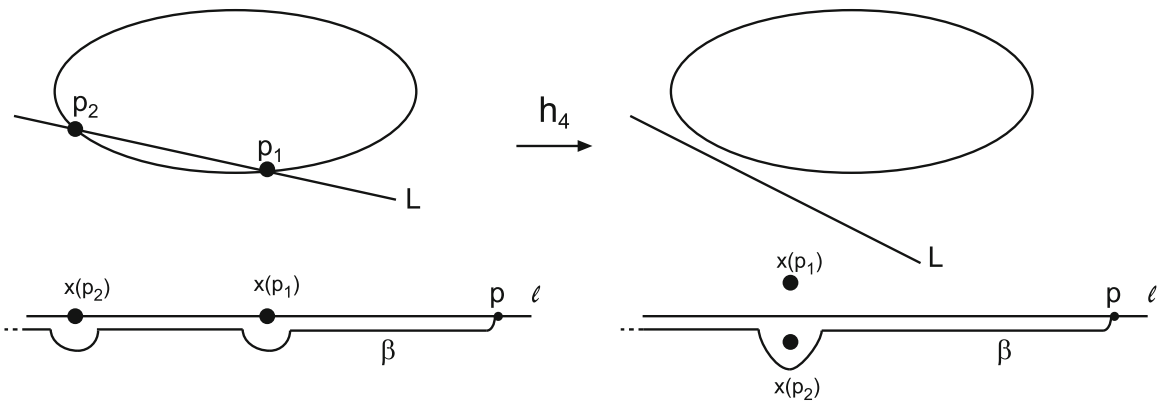

(a)

(b)

Fig. 14 A local model for a complex rotation and its effect on the path $\beta$

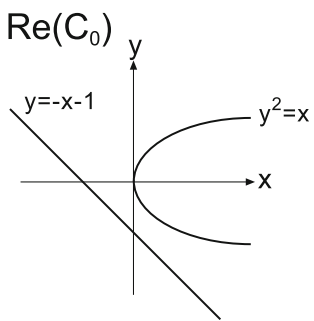

(a)

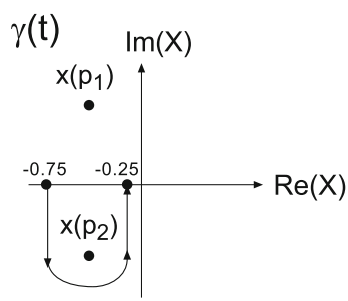

(b)

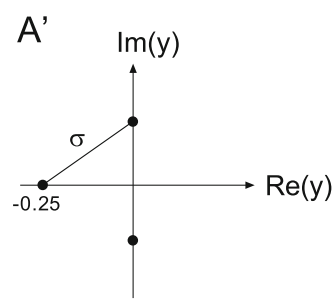

(c)

Fig. 15 A local model for a complex intersection between a line and a conic

analyze the situation after the rotation of $L$ (see Fig. 14b), recall the following setting from [16]: let $C_{0}=\left\{\left(y^{2}-x\right)(y+x+1)=0\right\}$ (see its real part in Fig. 15a). Define the projections $\pi_{1}, \pi_{2}: C_{0} \rightarrow \mathbb{C}$ by $\pi_{1}(x, y)=x, \pi_{2}(x, y)=y$. Denote by $p_{1}, p_{2}$ the complex intersection points of the curves $y^{2}=x$ and $y=-x-1$. Define:

$$
\begin{gathered}
x_{0}=-\frac{1}{4}, A=\pi_{2}\left(\pi_{1}^{-1}\left(x_{0}\right)\right)=\left\{ \pm \frac{1}{2} i,-\frac{3}{4}\right\}, \\
x_{1}=-\frac{3}{4}, A^{\prime}=\pi_{2}\left(\pi_{1}^{-1}\left(x_{1}\right)\right)=\left\{ \pm \frac{\sqrt{3}}{2} i,-\frac{1}{4}\right\} .
\end{gathered}
$$

Let $\gamma(t), 0 \leq t \leq 1$, be a curve starting at $x_{1}$, ending at $x_{0}$ and surrounding $x\left(p_{2}\right)$ from below (see Fig. 15b). Let $D$ be a disk in the $y$-axis such that $A, A^{\prime} \subset D$ and denote by $\psi_{\gamma}$ the Lefschetz diffeomorphism induced by $\gamma(t)$. Let $\sigma$ be the segment connecting $-\frac{1}{4}$ and $\frac{\sqrt{3} i}{2}$ in $A^{\prime}$, see Fig. 15c. Then, by [16, Corollary 2.2], $\psi_{\gamma}=(H(\sigma))^{2}$, where $H(\sigma)$ is the half-twist induced by the path $\sigma$.

It is now clear that if we rotate the two complex points by $90^{\circ}$ counterclockwise in the fiber (in order to obtain a real model for the fiber), we get, in both cases, that the same braid is applied, as needed.

After we have proved these two lemmata, we are ready to finish the proof of Proposition 2.18(2). 
Lemma 4.3 Let $\mathcal{A}$ be a real $C L$ arrangement with one conic such that $\beta(\mathcal{A})=0$. Assume that $\mathcal{A}$ can be presented as a union $\mathcal{A}=\mathcal{A}^{\prime} \cup L$, where $\mathcal{A}^{\prime}$ is a real $C L$ arrangement with a conjugation-free presentation of $\pi_{1}\left(\mathbb{C}^{2}-\mathcal{A}^{\prime}, u\right)$ for real every basepoint $u \in \ell-N$, and $L \notin \mathcal{A}^{\prime}$ is a real line that passes through at most one singular point $p$ of $\mathcal{A}^{\prime}$. Then, in this class of real CL arrangements, the operation $h_{3}$ preserves the conjugation-free property for $\mathcal{A}$, i.e. $\pi_{1}\left(\mathbb{C}^{2}-\mathcal{A}, u\right)$ has a conjugationfree geometric presentation for every real basepoint $u \in \ell-N$.

As in Remark 3.7, note that this lemma is not so restrictive as it seems in a first glance. Taking a real CL arrangement with one conic (ellipse or hyperbola) and some lines such that the only singular points (with respect to the projection) are nodes and the branch points of the conic, the affine fundamental group is abelian and as the relations induced by the branch points are equalities between generators, this group has a conjugation-free presentation. Thus, according to the lemma, one can add lines passing through at most one singular point and get the desired real CL arrangement (see also Sect. 6 for more details).

Proof Note that we can rotate the line $L$ around the point $p$ until it is almost vertical to the reference line $\ell$ by operations $h_{1}$ and $h_{2}$. We remind again that whenever we refer to the basepoint $u$, we implicitly assume that $u$ is real. We divide the proof to a number of steps.

In the first step, we prove this lemma for only two basepoints $u \in \ell-N$ (where $N$ is the set of the projections of the singular points). In the other steps, we emphasize the changes we have to make in order to modify the proof of Proposition 2.18(1), so that we can use the corresponding steps as in the proof of that proposition.

We prove this lemma for the case where the conic is either an ellipse or a hyperbola (recall that we do not include a parabola in our real CL arrangements, see Remark 2.5(2)).

Let $p^{\prime}$ (resp. $\alpha$ ) be the singular point on $L$ with the minimal (resp. maximal) real value of its $x$-coordinate. Note that here $\alpha$ is not necessarily a real point (i.e. its coordinates can be complex), as can be seen in case (1) in the proof of Step 4.4.

Step 4.4 Lemma 4.3 holds for $u=x(\alpha)+\varepsilon$ or $u=x\left(p^{\prime}\right)-\varepsilon$.

Proof We divide the proof into two cases. The first case covers the cases where either $C$ is an ellipse and $p$ is to the left of its two branch points, or $C$ is a hyperbola and $p$ is between the two branch points. The second case covers the additional two cases.

Case (1): This case deals with the case where either $C$ is a hyperbola and $p$ is between the two branch points, or $C$ is an ellipse and $p$ is to the left of its two branch points (if $p$ is to the right of the two branch points, we can reflect the arrangement with respect to the $y$-axis and proceed as follows). We can rotate the line $L$ to be a line with a very negative slope, almost vertical to the reference line $\ell$. Thus we get the arrangement in Fig. 16a or the arrangement in Fig. 16b. We will work with the arrangement in Fig. 16a for notational reasons. However, the only thing that matters is that the line $L$ intersects the conic $C$ in two complex intersection points $\alpha, \alpha^{\prime}$.

As proved in Lemma 4.2, the rotation of the line $L$ preserves the conjugation-free property (if it exists before or after the rotation; recall that Lemma 4.2 has a local 


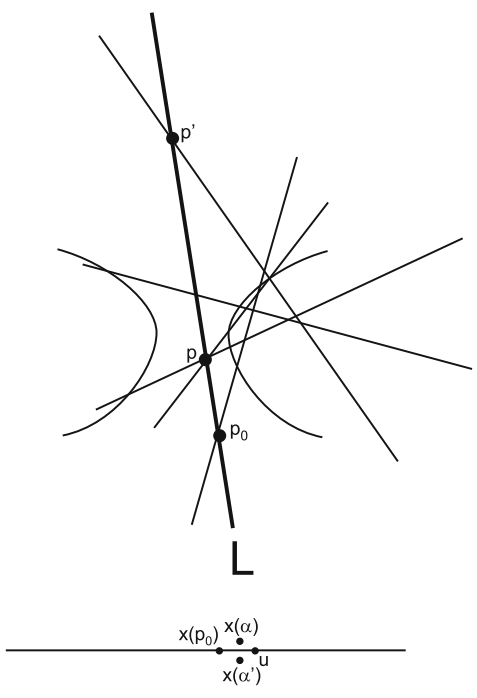

(a)

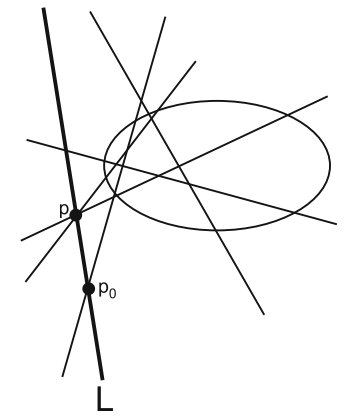

(b)

Fig. 16 First case: an illustration for the real part of $\mathcal{A}=\mathcal{A}^{\prime} \cup L$ with a hyperbola (a) or with an ellipse (b)

nature, i.e. it does not matter if $C$ is either an ellipse or a hyperbola). Let $p_{0}$ be the lowest real singular point on the line $L$ (with respect to the $y$-coordinate). As the slope of $L$ is very negative, we can assume that $x\left(p_{0}\right)<x(\alpha)$ (by computation) and we choose a basepoint $u \in \ell$ such that $u=x(\alpha)+\varepsilon$. We first prove that the induced relations of all the singular points on $L$ have no conjugations.

Note that the relations in $\pi_{1}\left(\mathbb{C}^{2}-\mathcal{A}, u\right)$ induced by the complex intersection points $\alpha, \alpha^{\prime}$ have no conjugations. Indeed, one can get conjugations if the path, going from the basepoint to the singular point in question, goes below other singular points. This is not the case here, as the points $\alpha, \alpha^{\prime}$ are the closest points to $u$.

Hence, the generator corresponding to the line $L$ commutes with the generators of the conic $C$. In the fiber over $u$ (i.e. the fiber $\mathbb{C}_{u}^{1}=\pi^{-1}(u)$ ) there are two complex points $y(\beta), y\left(\beta^{\prime}\right)$, where $C \cap \pi^{-1}(u)=\left\{\beta, \beta^{\prime}\right\}$. We consider the model of this fiber as a real fiber by rotating these complex points by $90^{\circ}$ counterclockwise; see Fig. 17a.

As for the relation induced by the point $p_{0}$, we have to apply the braid $(H(\sigma))^{2}$ on the initial skeleton of $p_{0}$, where $\sigma$ is drawn in Fig. 17b (see the proof of Lemma 4.2), and then rotating the complex points by $90^{\circ}$ counterclockwise. The resulting skeleton is presented in Fig. 17c and since the generator corresponding to $L$ commutes with the generators corresponding to the hyperbola, the induced relation has no conjugations. A similar computation works for all the other intersection points on $L$. Thus, we proved that all the intersection points on $L$ induce relations without conjugations.

Note that the singular points which are to the right of $u$ already appeared in $\mathcal{A}^{\prime}$ and thus their associated skeletons are the same (when increasing the corresponding indices by 1). Let $p^{\prime}$ be the highest intersection point on $L$. 


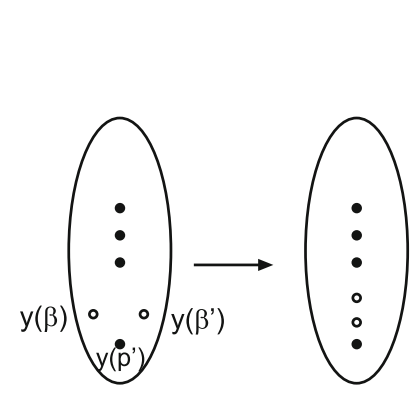

(a)

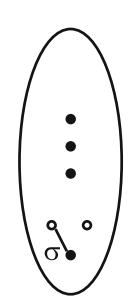

(b)

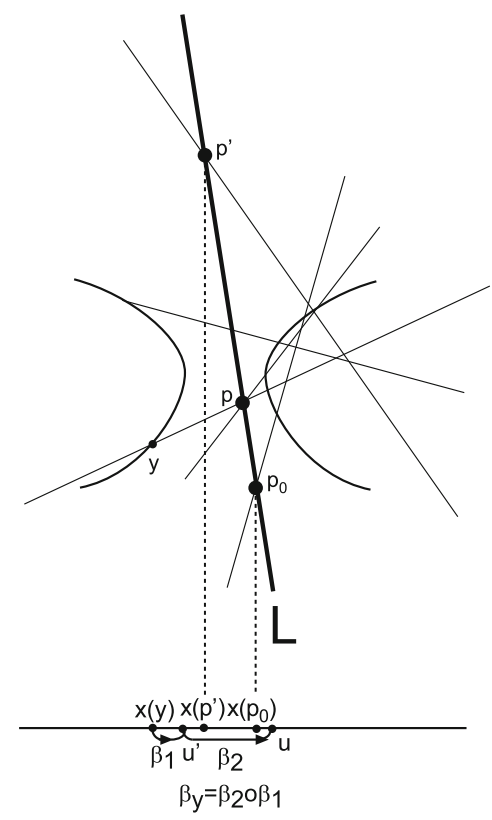

(d) (c)

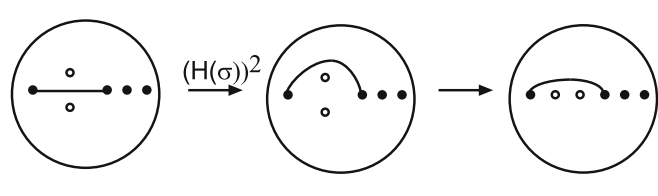

Fig. 17 Notations for computing the relations induced by the singular points on $L(\mathbf{a}-\mathbf{c})$ and for the proof that the relations induced by the singular points to the left of $L$ have no conjugations (d)

We now prove that all the skeletons of the singular points to the left of $p^{\prime}$ are the same when computed in $\mathcal{A}$ and in $\mathcal{A}^{\prime}$, besides the fact that the indices of the points of the skeletons in $\mathcal{A}$ are increased by 1 (this is due to the fact that $L$ is numbered as 1 in the fiber over $u$ ). Once we prove that, we can use the fact that $\pi_{1}\left(\mathbb{C}^{2}-\mathcal{A}^{\prime}, u\right)$ is conjugation-free and the methods outlined in part (II) of the proof of Step 3.8 to simplify these relations and to prove that $\pi_{1}\left(\mathbb{C}^{2}-\mathcal{A}, u\right)$ has a conjugation-free geometric presentation.

In order to prove that, we need additional notations. Numerate the intersection points on $L$, starting from the highest real intersection point on $L$, by $p_{u}=p^{\prime}, \ldots, p_{v}=p, \ldots, p_{1}, p_{0}, \alpha, \alpha^{\prime}$ (note that all the intersection points are nodes except for $p_{v}$ which is an intersection point of multiplicity $m$ ) and denote their corresponding Lefschetz pairs by

- $s_{u}=[n-3, n-2], \ldots, s_{v+1}=[v+m, v+m+1]$,

- $s_{v}=[v+1, v+m]$,

- $s_{v-1}=[v, v+1], \ldots, s_{0}=[1,2], s_{\alpha}, s_{\alpha^{\prime}}$,

where $n-2$ is the number of lines in $\mathcal{A}$ (recall that we get two additional generators from the hyperbola).

Let $u^{\prime}=x\left(p^{\prime}\right)-\varepsilon$ (see Fig. 17d). For every singular point $y \in \mathcal{A}$ which is to the left of $u^{\prime}$, let $s_{y}$ be the initial skeleton of $y$. We now consider the braids which are applied on $s_{y}$ while going along a path $\beta_{y}$ starting at $x(y)+\varepsilon$ and ending at $u$. This 
path can be divided into two parts: the first part $\beta_{1}$ which starts at $x(y)+\varepsilon$ and ends at $u^{\prime}$ and the second part $\beta_{2}$ which starts at $u^{\prime}$ and ends at $u$.

Let $\left(s_{y}^{\prime}\right)_{1}$ (resp. $\left.\left(s_{y}^{\prime}\right)_{2}\right)$ be the skeleton of $y$ after we apply on it the braids induced by $\beta_{1}$ (resp. $\beta_{y}$ ) in the arrangement $\mathcal{A}^{\prime}$ and let $\left(s_{y}\right)_{1}$ (resp. $\left.\left(s_{y}\right)_{2}\right)$ be the corresponding skeleton after we apply on it the braids induced by $\beta_{1}$ (resp. $\beta_{y}$ ) in the arrangement $\mathcal{A}$.

Note that the braid induced by the path $\beta_{2}$ in $\mathcal{A}^{\prime}$ is

$$
\Delta\langle v+1, v+m-1\rangle,
$$

as $p \in \mathcal{A}^{\prime}$ is the only intersection point (with multiplicity $m-1$ ) in the section $\left\{m \in \mathbb{C}^{2}: u^{\prime}<x(m)<u\right\}$. Now, the braid induced by the path $\beta_{2}$ in $\mathcal{A}$ is:

$$
B_{2} \doteq \Delta\langle n-3, n-2\rangle \cdots \Delta\langle v+1, v+m\rangle \cdots \Delta\langle 2,3\rangle \Delta\langle 1,2\rangle(H(\sigma))^{2} .
$$

Note that the fibers over $x(y)+\varepsilon$ in $\mathcal{A}$ and in $\mathcal{A}^{\prime}$ differ only by one point, as there is an additional point in the fiber of $\mathcal{A}$, being the highest point (numbered as $n$ ) which represents the line $L$. Note also that the skeletons $\left(s_{y}^{\prime}\right)_{1}$ and $\left(s_{y}\right)_{1}$ are the same, as the line $L$ does not intersect the other components in the section between $x(y)$ and $u^{\prime}$. The only difference is that the fiber over $u^{\prime}$ in $\mathcal{A}$ has an additional point (being the highest point which represents the line $L$ ), and the skeleton $\left(s_{y}\right)_{1}$ does not go around this point.

Now, when applying $B_{2}$ on $\left(s_{y}\right)_{1}$, recall that $\beta_{2}$ is a path beneath all the intersection points of $L$ (that is, there are no intersection points on $L$ outside the section $\left.\left\{a \in \mathbb{C}^{2}: u^{\prime}<x(a)<u\right\}\right)$. Since $L$ is almost vertical with a negative slope, the braid $B_{2}$ induces on the point numbered as $n$ in $\mathcal{A}$ (in the fiber over $u^{\prime}$ ) the motion $\delta_{n}$, that is, the point $n$ performs a counterclockwise $180^{\circ}$ rotation, eventually turning into the point numbered as 1 . Moreover, as the point $n$ (in the fiber over $u^{\prime}$ ) is not involved in the skeleton $\left(s_{y}\right)_{1}$, the only braid that does affect $\left(s_{y}\right)_{1}$ is $\Delta\langle v+1, v+m\rangle$. At the stage of applying $\Delta\langle v+1, v+m\rangle$ (in $\mathcal{A}$ ), the point that was numbered as $n$ is then numbered as $v+m$ and is beneath the skeleton $\left(s_{y}\right)_{1}$. The braid $\Delta\langle v+1, v+m\rangle$ sends the point $v+m$ to the point $v+1$ and the effect on the skeleton itself is as the effect of $\Delta\langle v+1, v+m-1\rangle$. This means that the skeletons $\left(s_{y}^{\prime}\right)_{2}$ and $\left(s_{y}\right)_{2}$ are the same, except for that all the indices in $s_{y}^{\prime \prime}$ are increased by 1 . As was indicated above, we can use the same simplification process with the method described in Part (II) of the proof of Step 3.8 to simplify all the relations.

As for the case when the basepoint of $\pi_{1}\left(\mathbb{C}^{2}-\mathcal{A}\right)$ is $u=x\left(p^{\prime}\right)-\varepsilon$, the proof follows the same arguments as above.

Case (2): This case covers the remaining two situations: either that $C$ is an ellipse and $p$ is between the two branch points or that $C$ is a hyperbola and $p$ is not between the two branch points. As in case (1), we first rotate the line $L$ to be almost vertical with a negative slope. In both situations, the line $L$ intersects $C$ in two real points and thus these two situations present the same behavior. After the rotation of $L$, we get the arrangement in Fig. 18a (though Fig. 18 presents the case of an ellipse rather than a hyperbola, the proof is independent of the model of $C$ in $\mathbb{R}^{2}$, since we do not use the fact that $C$ is an ellipse, but rather the fact that $L$ intersects $C$ in two real points). 


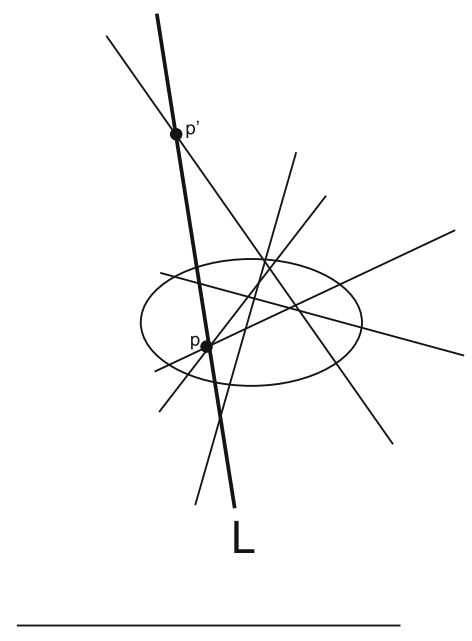

(a)

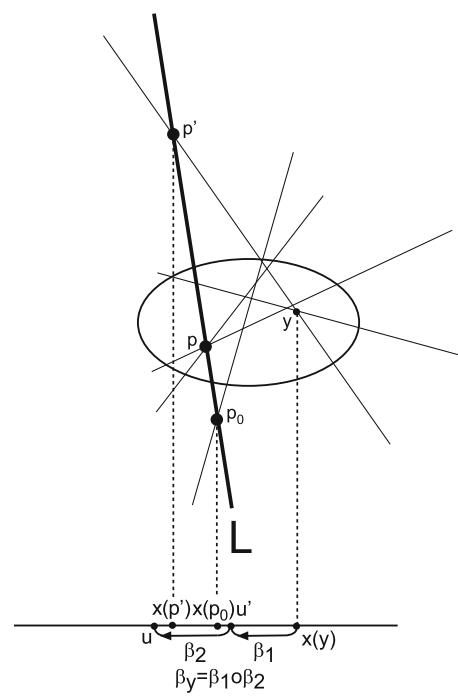

(b)

Fig. 18 Second case: Part a is an illustration for the real part of $\mathcal{A}=\mathcal{A}^{\prime} \cup L$ for an ellipse. Part b depicts notations for the proof of the claim that the relations induced by the singular points to the right of $L$ have no conjugations

Let $p^{\prime}$ be the highest intersection point of $L$ (with respect to the $y$-coordinate) and choose $u \in \ell$ such that $u=x\left(p^{\prime}\right)-\varepsilon$, where $0<\varepsilon \ll 1$. We choose the basepoint of $\pi_{1}\left(\mathbb{C}^{2}-\mathcal{A}\right)$ to be $u$. Numerate the intersection points on $L$, starting from the lowest point on $L$, by $p_{0}, p_{1}, \ldots, p_{v}=p, \ldots, p_{u}=p^{\prime}$ (note that all the points $p_{i}$ are nodes except for $p_{v}$ which is an intersection point of multiplicity $m$ ) and denote their corresponding Lefschetz pairs by

- $s_{0}=[1,2], s_{1}=[2,3], \ldots, s_{v-1}=[v, v+1]$,

- $s_{v}=[v+1, v+m]$,

- $s_{v+1}=[v+m, v+m+1], \ldots, s_{u}=[n-1, n]$,

where $n-2$ is the number of lines in $\mathcal{A}$ (recall that we get two additional generators from the ellipse). Define $u^{\prime} \in \ell$ as $u^{\prime}=x\left(p_{0}\right)+\varepsilon$.

We will prove that all the skeletons of the singular points to the left of $u$ or to the right of $u^{\prime}$ are exactly the same when being computed in $\mathcal{A}$ or in $\mathcal{A}^{\prime}$. Once we prove that, combined with the fact that all the relations induced by the singular points on $L$ have no conjugations, then we can use the fact that $\pi_{1}\left(\mathbb{C}^{2}-\mathcal{A}^{\prime}, u\right)$ is conjugation-free and the method described in Part (II) of the proof of Step 3.8 to simplify these relations and to prove that $\pi_{1}\left(\mathbb{C}^{2}-\mathcal{A}, u\right)$ has a conjugation-free geometric presentation.

However, the proof now follows exactly the same arguments described in Step 3.8 (indeed, the notations described in Fig. 18b work also for that step), and thus we refer the reader to there. As for the case when the basepoint of $\pi_{1}\left(\mathbb{C}^{2}-\mathcal{A}\right)$ is $u=x\left(p_{0}\right)+\varepsilon$, the proof follows the same arguments as above (see e.g. Step 3.9). 
We now prove that moving the basepoint below a projection of a branch point preserves the conjugation-free property.

Step 4.5 Let $b$ be a branch point of a conic, and denote $u=x(b)+\varepsilon, u^{\prime}=x(b)-\varepsilon$. If $\pi_{1}\left(\mathbb{C}^{2}-\mathcal{A}, u\right)$ is conjugation-free, then $\pi_{1}\left(\mathbb{C}^{2}-\mathcal{A}, u^{\prime}\right)$ is also conjugation-free.

Proof Without loss of generality, we can assume that the fiber over $u$ contains $n$ real points, where the points numbered as $c, c+1$ represent the conic, and thus the fiber over $u^{\prime}$ contains $n-2$ real points and 2 complex points: indeed, the points numbered as $c, c+1$ perform a $90^{\circ}$ counterclockwise rotation when passing from $u$ to $u^{\prime}$. This rotation describes the result of the isomorphism $\pi_{1}\left(\mathbb{C}^{2}-\mathcal{A}, u\right) \rightarrow \pi_{1}\left(\mathbb{C}^{2}-\mathcal{A}, u^{\prime}\right)$ at the level of the skeletons. However, when we work with a real model $(D, K)$ of the braid group (using Definition 2.8), we can perform any diffeomophism equivalent to the identity and get the same presentation by means of generators and relations. Thus, when taking the basepoint to be $u^{\prime}$, we can perform on every skeleton a $90^{\circ}$ clockwise rotation on the points numbered as $c, c+1$. This diffeomorphism is the identity at the level of the generators associated to the conic in $\pi_{1}\left(\mathbb{C}^{2}-\mathcal{A}, u^{\prime}\right)$, and therefore we can use the same simplification process as was done in $\pi_{1}\left(\mathbb{C}^{2}-\mathcal{A}, u\right)$ for proving that $\pi_{1}\left(\mathbb{C}^{2}-\mathcal{A}, u^{\prime}\right)$ is conjugation-free.

We now prove that $\pi_{1}\left(\mathbb{C}^{2}-\mathcal{A}, u\right)$ has a conjugation-free geometric presentation for every basepoint $u$ that belongs to one of the following domains:

- $D_{1}=\left\{u \in \ell-N: x\left(p^{\prime}\right)<u<x(\alpha)\right\}$.

- $D_{2}=\{u \in \ell-N: x(\alpha)<u\}$.

- $D_{3}=\left\{u \in \ell-N: x\left(p^{\prime}\right)>u\right\}$.

Step 4.6 For $u \in D_{1}, \pi_{1}\left(\mathbb{C}^{2}-\mathcal{A}, u\right)$ has a conjugation-free geometric presentation.

Proof The proof is the same as the proof of Step 3.10.

Before proving that $\pi_{1}\left(\mathbb{C}^{2}-\mathcal{A}, u\right)$ has a conjugation-free geometric presentation for the domains $D_{2}$ and $D_{3}$, we want to examine what are the implications that $\pi_{1}\left(\mathbb{C}^{2}-\mathcal{A}, u\right)$ has a conjugation-free geometric presentation for a certain basepoint $u$. In particular, this means that whenever we have a relation in the presentation of $\pi_{1}\left(\mathbb{C}^{2}-\mathcal{A}, u\right)$, written by the geometric generators which has conjugations of the generators, these conjugations can be removed.

One important implication of the conjugation-free property is that while the conic induces two geometric generators $x_{1}, x_{2}$ in $\pi_{1}\left(\mathbb{C}^{2}-\mathcal{A}, u\right)$, the conjugation-free property implies that the relations, inducing by both branch points, are $x_{1}=x_{2}$. Thus, we can say that not only the conic contributes only one generator in the presentation of $\pi_{1}\left(\mathbb{C}^{2}-\mathcal{A}, u\right)$, denoted by $x$, but that there are no other relations induced by the branch points (apart of $x_{1}=x_{2}$ ). Note that if the presentation is not conjugation-free, we may get new relations from the branch points which are not $x_{1}=x_{2}$ (see e.g. the third step in the proof of [15, Theorem 4.2]).

Recall that we assume that $\beta(\mathcal{A})=0$ and $\operatorname{deg}(\mathcal{A})=n$.

Step 4.7 Assume that $\pi_{1}\left(\mathbb{C}^{2}-\mathcal{A}, u\right)$ has a conjugation-free geometric presentation. Let $\Gamma_{k_{1}}, \ldots, \Gamma_{k_{n-2}}$ be the geometric generators associated to the $n-2$ lines of $\mathcal{A}$, and $x$ is the generator of the conic. Then $\left[x, \Gamma_{k_{i}}\right]=e$ for all $1 \leq i \leq n-2$. 
Proof First, note that if the conic $C$ intersects a line $L_{\alpha}$ transversally at two nodes, then $\left[x, \Gamma_{\alpha}\right]=e$, due to the conjugation-free property. Thus we assume that there is at least one multiple point in $\mathcal{A}$ which the conic passes through it. Note that $\beta(\mathcal{A})=0$ implies that the graph $G(\mathcal{A})$ is a forest. We look at the forest $G(\mathcal{A})$ and we start from a leaf, assuming that the conic passes through the intersection point of multiplicity $m+1$ that corresponds to this leaf (if not, move to its direct ancestor, i.e. to the next step in the proof).

In this case, the relations induced by this point are:

$$
\Gamma_{i_{m}} \cdots \Gamma_{i_{1}} x=\Gamma_{i_{m-1}} \cdots \Gamma_{i_{1}} x \Gamma_{i_{m}}=\cdots=\Gamma_{i_{1}} x \Gamma_{i_{m}} \cdots \Gamma_{i_{2}}=x \Gamma_{i_{m}} \cdots \Gamma_{i_{1}}
$$

where $\Gamma_{i_{j}}, 1 \leq j \leq m$, are the geometric generators associated to the lines $L_{i_{j}}$ which pass through this multiple point. Note that there are no conjugations in the relations, since the presentation is a conjugation-free. Since we are dealing with a leaf, all the lines (except maybe for one, which corresponds to the edge connected to its direct ancestor) intersect the conic also in a node. Numerate the lines in such a way that $L_{i_{1}}$ is the line that possibly does not intersect the conic in a simple point. Again, since the group has a conjugation-free geometric presentation, we have the following relations, induced by these simple points:

$$
\left[x, \Gamma_{i_{j}}\right]=e, \text { for all } 2 \leq j \leq m .
$$

Therefore, from relations (11) and (12), one can easily deduce that

$$
\left[x, \Gamma_{i_{1}}\right]=e .
$$

Indeed, using relations (12) and the equality $\Gamma_{i_{m}} \cdots \Gamma_{i_{1}} x=x \Gamma_{i_{m}} \cdots \Gamma_{i_{1}}$ (left hand side and right hand side of relations (11)), we have $\left[x, \Gamma_{i_{1}}\right]=e$ as needed.

With this data, we can proceed to the immediate upper level of the tree, which means that we proceed to the second multiple point that is on $L_{i_{1}} \cap C$ (if it exists).

Now, we do the same process as above to the new level, as this point can now be treated as a "leaf", i.e. with the same properties regarding the relations in the fundamental group. In this way, we go over all the vertices of the graph that the conic passes through the corresponding multiple points.

Remark 4.8 Note that the essential data that we have used during the proof of Step 4.7 is that there is a vertex (a leaf, to be exact) in the graph, that all the lines, except maybe for one, that passes through it, intersect the conic in a node. We will use that property in order to generalize Step 4.7 for other arrangements whose graph may contain a cycle.

We use Step 4.7 in order to prove that if $u$ is in the domains $D_{2}$ and $D_{3}, \pi_{1}\left(\mathbb{C}^{2}-\mathcal{A}, u\right)$ has a conjugation-free geometric presentation too.

Step 4.9 For $u \in D_{2}=\{u \in \ell-N: x(\alpha)<u\}$, $\pi_{1}\left(\mathbb{C}^{2}-\mathcal{A}, u\right)$ has a conjugationfree geometric presentation. 


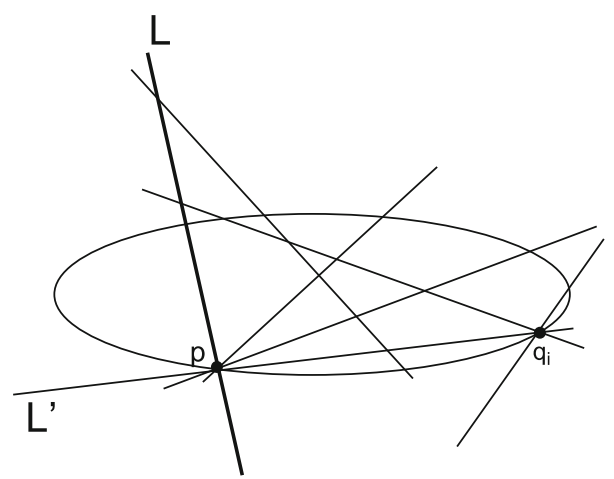

Fig. 19 The case where the conic and a line $L^{\prime}$, which pass through $p$, pass through another singular point $q_{i}$ too

Proof The proof of this step is based on the following three claims:

(1) All the skeletons induced by the intersection points not on $L$ are the same in $\mathcal{A}$, as if they were computed in $\mathcal{A}^{\prime}$, except for that all the indices in $\mathcal{A}$ are increased by 1 .

(2) All the relations induced by the nodes on $L$ have no conjugations.

(3) The relation induced by $p \in L$ has no conjugations.

The proofs of claims (1) and (2) are exactly the same as the proofs of the corresponding claims in Step 3.11. However, for claim (3), we have to perform some changes in the proof. As in Step 3.11, the proof is by induction on the location of $u$, and we use the same notations from that step. Explicitly, let $q_{1}, \ldots, q_{k}$ be the singular points in the domain $D_{2}$, and we assume that $u=x\left(q_{i}\right)-\varepsilon, u^{\prime}=x\left(q_{i}\right)+\varepsilon$, when we know, by the induction hypothesis that the conjugation-free property holds for $u \in\left\{m \in \ell-N: x\left(q_{1}\right)-\varepsilon<m<x\left(q_{i}\right)\right\}$. Recall that the Lefschez pair of the point $p \in L$ (in the fiber above $x(p)+\varepsilon$ ) is $[v+1, v+m]$.

When the basepoint is $u$, then the relation induced by the point $p \in L$ is:

$$
\left[\Gamma_{1}, \Gamma_{B_{u}(v+2)}, \Gamma_{B_{u}(v+3)}, \ldots, \Gamma_{B_{u}(v+m)}\right]=e,
$$

due to the conjugation-free property of $\pi_{1}\left(\mathbb{C}^{2}-\mathcal{A}, u\right)$. Note that when going to the basepoint $u^{\prime}$, the induced isomorphism is $f_{3}^{\prime}$ as described in Eq. (6). However, now we should note that $f_{3}^{\prime}$ can affect at most two of the generators $\Gamma_{B_{u}(x)}, v+2 \leq x \leq v+m$. This is different from the situation in line arrangements, where it is impossible that two different lines will pass both through $p$ and $q_{i}$. On the other hand, in CL arrangements, the conic and a line $L^{\prime}$ can both pass through $p$ and $q_{i}$ (see Fig. 19). Hence, two different geometric generators, in the relation induced by $p$, might be affected by $f_{3}^{\prime}$.

We now divide the proof into three cases: when $f_{3}^{\prime}$ does not affect any of the generators $\Gamma_{B_{u}(x)}$, when it affects only one of them and when it affects two of them. As for the first two cases, the proof is the same as the proof of cases (1) and (2) of claim (3) in the proof of Step 3.11. We now deal with the third case. 
Recall that the conic contributes two generators to $\pi_{1}\left(\mathbb{C}^{2}-\mathcal{A}, u\right)$, denoted by $x_{1}, x_{2}$. Assume that in the fiber over $u$, the local numeration of the components intersecting in $q_{i}$ is $a, a+1, \ldots, a+s=b$, where $1<a$ (since the line $L$ is numbered as 1 in the fiber over $u$ ). Without loss of generality, assume that over $u$, it is the generator $x_{1}$ that participates in the relation (13), such that $x_{1}$ is equal to $\Gamma_{a+t}$, where $0 \leq t \leq s$. Since $\pi_{1}\left(\mathbb{C}^{2}-\mathcal{A}, u\right)$ is conjugation-free, we know that $x_{1}=x_{2}$ in $\pi_{1}\left(\mathbb{C}^{2}-\mathcal{A}, u\right)$. Moreover, by Step 4.7, we know that $\left[\Gamma_{i}, x_{2}\right]=e$ for any generator $\Gamma_{i}$ associated to a line in $\mathcal{A}$.

Thus, in $\pi_{1}\left(\mathbb{C}^{2}-\mathcal{A}, u^{\prime}\right)$,

$$
f_{3}^{\prime}\left(x_{1}\right)=f_{3}^{\prime}\left(\Gamma_{a+t}\right) \doteq \Gamma_{b}^{\prime} \cdots \Gamma_{b-t+1}^{\prime} \Gamma_{b-t}^{\prime} \Gamma_{b-t+1}^{\prime-1} \cdots \Gamma_{b}^{\prime-1}
$$

and $f_{3}^{\prime}\left(x_{2}\right)=x_{2}^{\prime}$. Therefore, the relation $x_{1}=x_{2}$ is mapped in $\pi_{1}\left(\mathbb{C}^{2}-\mathcal{A}, u^{\prime}\right)$ to $f_{3}^{\prime}\left(x_{1}\right)=f_{3}^{\prime}\left(x_{2}\right)$, which is:

$$
\Gamma_{b}^{\prime} \cdots \Gamma_{b-t+1}^{\prime} \Gamma_{b-t}^{\prime} \Gamma_{b-t+1}^{\prime-1} \cdots \Gamma_{b}^{\prime-1}=x_{2}^{\prime}
$$

Now, in $\pi_{1}\left(\mathbb{C}^{2}-\mathcal{A}, u\right)$, we know that $\left[\Gamma_{a}, x_{2}\right]=e$. Thus, in $\pi_{1}\left(\mathbb{C}^{2}-\mathcal{A}, u^{\prime}\right)$ we have that

$$
\left[f_{3}^{\prime}\left(\Gamma_{a}\right), f_{3}^{\prime}\left(x_{2}\right)\right]=e \rightarrow\left[\Gamma_{b}^{\prime}, x_{2}^{\prime}\right]=e .
$$

Also, the existing relation $\left[\Gamma_{a+1}, x_{2}\right]=e$ in $\pi_{1}\left(\mathbb{C}^{2}-\mathcal{A}, u\right)$ is transformed in $\pi_{1}\left(\mathbb{C}^{2}-\right.$ $\left.\mathcal{A}, u^{\prime}\right)$ to:

$$
\left[f_{3}^{\prime}\left(\Gamma_{a+1}\right), f_{3}^{\prime}\left(x_{2}\right)\right]=e \rightarrow\left[\Gamma_{b}^{\prime} \Gamma_{b-1}^{\prime} \Gamma_{b}^{\prime-1}, x_{2}^{\prime}\right]=e \stackrel{\left[\Gamma_{b}^{\prime}, x_{2}^{\prime}\right]=e}{\rightarrow}\left[\Gamma_{b-1}^{\prime}, x_{2}^{\prime}\right]=e
$$

In the same way, we get that $\left[\Gamma_{j}^{\prime}, x_{2}^{\prime}\right]=e$ for every $b-t+1 \leq j \leq b$ in $\pi_{1}\left(\mathbb{C}^{2}-\mathcal{A}, u^{\prime}\right)$. Thus, relation (15) is simplified to $\Gamma_{b-t}^{\prime}=x_{2}^{\prime}$ and therefore, $e=\left[\Gamma_{j}^{\prime}, x_{2}^{\prime}\right]=\left[\Gamma_{j}^{\prime}, \Gamma_{b-t}^{\prime}\right]$ for any $b-t+1 \leq j \leq b$. Thus, by Eq. (14),

$$
f_{3}^{\prime}\left(\Gamma_{a+t}\right)=\Gamma_{b-t}^{\prime}
$$

Now, we deal with the behavior of the generator associated to the line $L^{\prime}$ passing through $p$ and $q_{i}$, under the isomorphism $f_{3}^{\prime}$. We would like to prove that if this generator is $\Gamma_{a+y}$, then $f_{3}^{\prime}\left(\Gamma_{a+y}\right)$ is a conjugation of $\Gamma_{b-y}^{\prime}$ by other geometric generators which commute with $\Gamma_{1}^{\prime}$.

We split the treatment into two cases.

If the line $L^{\prime}$ is numbered over $u$ as $a^{\prime} \doteq a+t-m$, for $m>0$, then

$$
f_{3}^{\prime}\left(\Gamma_{a+t-m}\right) \doteq \Gamma_{b}^{\prime} \cdots \Gamma_{b-t+m+1}^{\prime} \Gamma_{b-t+m}^{\prime} \Gamma_{b-t+m+1}^{\prime-1} \cdots \Gamma_{b}^{\prime-1}
$$

The conjugating generators of $\Gamma_{b-t+m}^{\prime}$ correspond to lines that intersect the line $L$ in nodes, and thus (by claim (2)) the generator $\Gamma_{1}^{\prime}$ commutes with all of them in $\pi_{1}\left(\mathbb{C}^{2}-\mathcal{A}, u^{\prime}\right)$. 
If this line is numbered over $u$ as $a^{\prime} \doteq a+t+m, m>0$, then

$$
f_{3}^{\prime}\left(\Gamma_{a+t+m}\right) \doteq \Gamma_{b}^{\prime} \cdots \Gamma_{b-t-m+1}^{\prime} \Gamma_{b-t-m}^{\prime} \Gamma_{b-t-m+1}^{\prime-1} \cdots \Gamma_{b}^{\prime-1}
$$

Note that one of the conjugating generators of $\Gamma_{b-t-m}^{\prime}$ is $\Gamma_{b-t}^{\prime}$. By Step 4.7, we know that $\left[\Gamma_{1}, x_{2}\right]=e$ in $\pi_{1}\left(\mathbb{C}^{2}-\mathcal{A}, u\right)$. Thus

$$
e=\left[f_{3}^{\prime}\left(\Gamma_{1}\right), f_{3}^{\prime}\left(x_{2}\right)\right]=\left[\Gamma_{1}^{\prime}, x_{2}^{\prime}\right] \stackrel{\Gamma_{b-t}^{\prime}=x_{2}^{\prime}}{=}\left[\Gamma_{1}^{\prime}, \Gamma_{b-t}^{\prime}\right]
$$

in $\pi_{1}\left(\mathbb{C}^{2}-\mathcal{A}, u^{\prime}\right)$. Therefore, using again the same argument above, $\Gamma_{1}^{\prime}$ commutes with all of conjugating elements of $\Gamma_{b-t+m}^{\prime}$.

To conclude, by Eq. (16), this means that relation (13) in $\pi_{1}\left(\mathbb{C}^{2}-\mathcal{A}, u^{\prime}\right)$ is turned to

$$
\left[\Gamma_{1}^{\prime}, \Gamma_{B_{u}(v+2)}^{\prime}, \Gamma_{B_{u}(v+3)}^{\prime}, \ldots, \Gamma_{a^{\prime}-1}^{\prime}, f_{3}^{\prime}\left(\Gamma_{a^{\prime}}^{\prime}\right), \Gamma_{a^{\prime}+1}^{\prime}, \ldots, \Gamma_{B_{u}(v+m)}^{\prime}\right]=e,
$$

where $f_{3}^{\prime}\left(\Gamma_{a^{\prime}}^{\prime}\right)$ equals to a conjugation of a geometric generator with generators, which commute with $\Gamma_{1}^{\prime}$. Therefore, we can now proceed exactly as described in the last paragraphs of the proof of Step 3.11.

The proof for $u \in D_{3}$ follows the same arguments as in the case for real line arrangements (see Step 3.12), and therefore, we have proved that $\pi_{1}\left(\mathbb{C}^{2}-\mathcal{A}, u\right.$ ) is conjugation-free for any $u \in \ell-N$.

This completes the proof of Proposition 2.18(2).

\section{Results and applications}

In the following two subsections, we would like to investigate the cases where $\beta(\mathcal{A})=$ 0 or $\beta(\mathcal{A})=1$ for a $\mathrm{CL}$ arrangement $\mathcal{A}$. The first subsection concentrates on the case where $\beta(\mathcal{A})=0$, examining the structure of the corresponding fundamental group. The second subsection concentrates on the case where $\beta(\mathcal{A})=1$, where the conic does not pass through all the multiple points which correspond to the vertices of the graph.

Moreover, we are especially interested to find the cases where the presentation and the structure of the affine fundamental group can be directly read from the graph (i.e. the lattice of the arrangement determines the fundamental group of its complement), so one of our aims in this section is to find out for which real CL arrangements, the fundamental group is either abelian or conjugation-free.

\subsection{The case of a graph with no cycles}

In this section, we prove Theorem 2.7. We first prove that the fundamental groups of the complements of these CL arrangements (with one conic), whose graph has no cycles, have a conjugation-free geometric presentation (see Proposition 5.1), and then we find out the structure of these fundamental groups. 


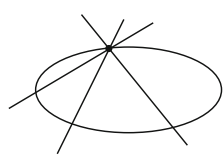

$\Rightarrow$
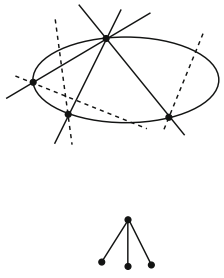

(b)
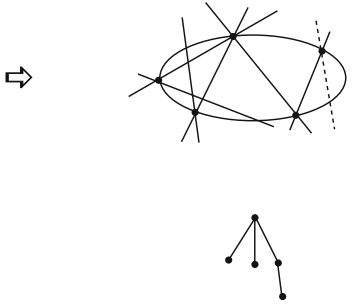

(c)

Fig. 20 An example for an inductive construction of the CL arrangement according to the graph (see Proposition 5.1): in step (a), we draw the conic and three more lines which create a multiple point on the conic, which corresponds to the tree's root. In step (b), we add three (dotted) lines creating three new multiple points, thus the associated graph of the arrangement is a tree with a root and three successors. In step (c), we add another (dotted) line creating a new multiple point, which corresponds to a new successor in the tree

Using Proposition 2.18(2) inductively, we have the following proposition:

Proposition 5.1 Let $\mathcal{A}$ be a real CL arrangement with one conic and $k$ lines. Suppose that $\beta(\mathcal{A})=0$. Then $\pi_{1}\left(\mathbb{C}^{2}-\mathcal{A}\right)$ has a conjugation-free geometric presentation.

Proof First, note that by Lemma 4.3, the conjugation-free geometric presentation is independent of the basepoint for this class of CL arrangements (assuming that the basepoint is real). Note that $\beta(\mathcal{A})=0$ implies that the graph $G(\mathcal{A})$ is a forest. Hence, the CL arrangement can be constructed inductively according to the graph (see an example in Fig. 20): first draw the conic and all the lines that do not contribute to the graph (i.e. the lines that do not pass through any multiple point). Obviously, the fundamental group of this arrangement has a conjugation-free geometric presentation for every real basepoint (since all the components are in general position, the fundamental group of the affine complement is abelian, due to [25]). Now start from the root of one of the trees, i.e. draw all the lines that correspond to the edges connected to this root (see Fig. 20a). By Proposition 2.18(2), the conjugation-free property is preserved. In the following steps, construct the rest of the arrangement by going to the direct successors of the tree's root, and drawing the corresponding lines (see Fig. 20b, c). Note that since at each step, we draw a single line passing through only one intersection point and there are no cycles in the resulting graph, the conjugation-free property is preserved. When comparing the resulting arrangement to the original one, the only lines that can be missing are lines that pass only through one multiple point. Thus adding these lines will again preserve the conjugation-free property.

Now, we do the same process to any other tree in the graph, if any. As this process is finite, we are done.

Now, we can prove Theorem 2.7 .

Proof of Theorem 2.7 By Step 4.7 (which now holds independently of the basepoint $u$ ), we can conclude that:

$$
\pi_{1}\left(\mathbb{C}^{2}-\mathcal{A}\right) \cong\langle x\rangle \oplus \pi_{1}\left(\mathbb{C}^{2}-(\mathcal{A}-C)\right)
$$


where $x$ is the generator of the conic. Thus, it remains to prove that $\pi_{1}\left(\mathbb{C}^{2}-(\mathcal{A}-C)\right)$ is isomorphic to a direct sum of free groups and a free abelian group. However, this is straight-forward, since $\beta(\mathcal{A})=0$ implies that $\beta(\mathcal{A}-C)=0$. Now, since $\mathcal{A}-C$ is an arrangement of lines, we can use Fan's result [14] that the fundamental group of an arrangement of lines whose graph has no cycles is a direct sum of free groups and a free abelian group.

Explicitly, this means that:

$$
\pi_{1}\left(\mathbb{C}^{2}-\mathcal{A}\right) \cong \mathbb{Z}^{r} \oplus \bigoplus_{i=1}^{p} \mathbb{F}_{m\left(a_{i}\right)-2} \oplus \bigoplus_{i=1}^{q} \mathbb{F}_{m\left(b_{i}\right)-1},
$$

where $r=k+2 p+q+1-\sum_{i=1}^{p} m\left(a_{i}\right)-\sum_{i=1}^{q} m\left(b_{i}\right)$.

As a result, we have an immediate corollary stating when the fundamental group is abelian.

Corollary 5.2 Let $\mathcal{A}$ be a real $C L$ arrangement with one conic $C$ with only branch points, nodes and triple points as singularities. Assume that $\beta(\mathcal{A})=0$ and all the triple points are on the conic. Then $\pi_{1}\left(\mathbb{C}^{2}-\mathcal{A}\right)$ is abelian.

There are some immediate consequences of Theorem 2.7, using the following decomposition theorem of Oka and Sakamoto [25]:

Theorem 5.3 (Oka-Sakamoto) Let $C_{1}$ and $C_{2}$ be algebraic plane curves in $\mathbb{C}^{2}$. Assume that the intersection $C_{1} \cap C_{2}$ consists of distinct $d_{1} \cdot d_{2}$ points, where $d_{i}(i=1,2)$ are the respective degrees of $C_{1}$ and $C_{2}$. Then:

$$
\pi_{1}\left(\mathbb{C}^{2}-\left(C_{1} \cup C_{2}\right)\right) \cong \pi_{1}\left(\mathbb{C}^{2}-C_{1}\right) \oplus \pi_{1}\left(\mathbb{C}^{2}-C_{2}\right) .
$$

We state the consequence for the case of CL arrangements with two conics, but the general case is straight-forward.

Corollary 5.4 Let $\mathcal{A}$ be a real CL arrangement with two conics and $k$ lines. Assume that the conics intersect each other transversally and $\beta(\mathcal{A})=0$. For $i=1,2$, let $V_{i}$ be the set of vertices of $G(\mathcal{A})$ whose corresponding points lie on the conic $C_{i}$. If $G(\mathcal{A})$ is a disjoint union of two graphs $G_{1}, G_{2}$ such that $V_{i} \subset G_{i}$, then $\pi_{1}\left(\mathbb{C}^{2}-\mathcal{A}\right)$ is a direct sum of free groups and a free abelian group.

Remark 5.5 The simplest case of a CL arrangement $\mathcal{A}$ with two conics, where we cannot apply Corollary 5.4, is presented in Fig. 21.

However, a direct calculation shows that $\pi_{1}\left(\mathbb{C}^{2}-\mathcal{A}\right)$ has a conjugation-free geometric presentation and thus abelian (e.g. $\left.\pi_{1}\left(\mathbb{C}^{2}-\mathcal{A}\right) \cong \mathbb{Z}^{5}\right)$. Therefore, it is reasonable to conjecture the following:

Conjecture 5.6 Let $\mathcal{A}$ be a real CL arrangement with $n$ conics and $k$ lines, where for each pair of conics, the two conics intersect each other transversally and neither a line nor another conic passes through those intersection points. Suppose that $\beta(\mathcal{A})=0$. Then $\pi_{1}\left(\mathbb{C}^{2}-\mathcal{A}\right)$ is a direct sum of free groups and a free abelian group. 


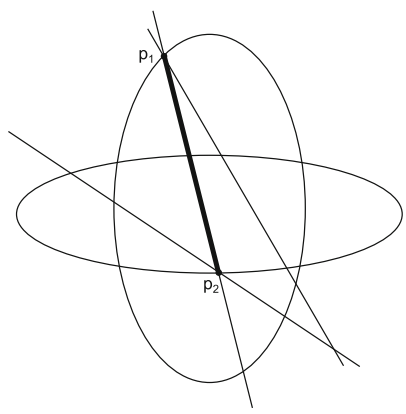

Fig. 21 The graph of the arrangement $\mathcal{A}$ is a single edge connecting the two vertices corresponding to the triple points $p_{1}, p_{2}$

We finish this section with the following conjecture for a general smooth plane curve:

Conjecture 5.7 Let $C$ be a smooth plane curve, $\mathcal{L}$ a real line arrangement, such that for each line $L \in \mathcal{L}, L$ intersects $C$ transversally in a real point. Define the graph $G(\mathcal{L} \cup C)$ as in the case of CL arrangements (see Definition 2.6). If $\beta(\mathcal{L} \cup C)=0$, then $\pi_{1}\left(\mathbb{C}^{2}-(\mathcal{L} \cup C)\right)$ is a direct sum of a free abelian group and free groups.

5.2 The case of a graph with one cycle, where the conic does not pass through all the vertices of the cycle

In the following section, we want to examine the case where $\beta(\mathcal{A})=1$ (for a CL arrangement with one conic), where the conic does not pass through all the multiple points corresponding to the vertices of the cycle (the case where the conic does pass through all the multiple points corresponding to the vertices of the cycle is studied in [15]). We prove that not only that the affine fundamental group of the complement has a conjugation-free geometric presentation, but that the generator of the conic commutes with all the other generators.

Proposition 5.8 Let $\mathcal{A}$ be a real CL arrangement with one conic $C$ such that:

(1) $\beta(\mathcal{A})=1$.

(2) There is a vertex $y \in G(\mathcal{A})$ such that $y$ is a vertex contained in the cycle of $G(\mathcal{A})$, $y \notin C$ and the two different edges exiting from $y$, which compose the cycle, are associated to two different lines in $\mathcal{A}$ (see Fig. 22), and both of them intersect the conic also in a node.

Then, $\pi_{1}\left(\mathbb{C}^{2}-\mathcal{A}\right)$ has a conjugation-free geometric presentation.

Proof Let $y \in \mathcal{A}$ be an intersection point satisfying Condition (2). There are $k$ lines $L_{1}, \ldots, L_{k}$ which pass through $y$; assume that $L_{1}, L_{2}$ are the lines which correspond to edges of the cycle of $G(\mathcal{A})$ (see Fig. 22) and $L_{3}, \ldots, L_{k}$ are not, otherwise there would be more than one cycle in $G(\mathcal{A})$. We can also assume that there are no other edges in the graph $G(\mathcal{A})$ exiting from $y$ (indeed, as will be explained later, by the 


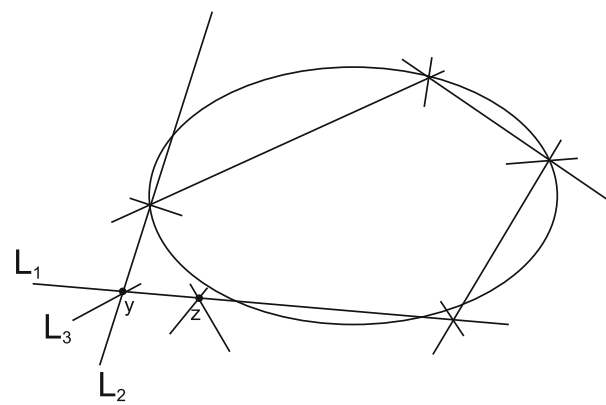

Fig. 22 The vertex $y$ is contained in the cycle of the graph, and satisfies Condition (2) and $L_{1}$ and $L_{2}$ are the lines correspond to two edges exiting from $y$, intersecting the conic also in a node. The vertex $z$ is contained in the cycle but it does not satisfy Condition (2), since the edges in $G(\mathcal{A})$ exiting from $z$ are associated to the same line $L_{1}$

same methods of Proposition 5.1, adding the corresponding lines will preserve the conjugation-free property).

We look at $\mathcal{A}^{\prime}=\mathcal{A}-\left\{L_{3}, \ldots, L_{k}\right\}$. Note that $\beta\left(\mathcal{A}^{\prime}\right)=0$ (since $y$ is a node in $\mathcal{A}^{\prime}$ and there is only one cycle in $G(\mathcal{A})$ ) and therefore $\pi_{1}\left(\mathbb{C}^{2}-\mathcal{A}^{\prime}\right)$ has a conjugation-free geometric presentation (by Proposition 5.1). Now, draw the line $L_{3}$ and rotate it till it is almost vertical to the reference line $\ell$. As was already proven, adding a line in almost vertical position does not affect the conjugation-free property. Now, by the same course of the proof of Lemma 4.3, we can still follow Steps 4.4, 4.5 and 4.6. The problem is Step 4.7 (proving that all the generators corresponding to the line commute with the generator $x$ corresponding to the conic), as it relies on the fact that $\beta(\mathcal{A})=0$. However, the proof of Step 4.7 starts with choosing one leaf of the graph $G(\mathcal{A})$ whose corresponding singular point is located on the conic $C$, and proving that all the generators, corresponding to the lines passing through that leaf, commute with $x$ (see Remark 4.8).

But if we choose the vertex corresponding to $y$ as that "leaf" here, we see that the same phenomenon happens here; indeed, $L_{1}$ and $L_{2}$ intersect $C$ also in a node, and therefore the corresponding generators commute with $x . L_{3}$ intersects $C$ in two nodes, and thus the corresponding generators commute with $x$. This means that for the ancestors of the vertex corresponding to $y$, there is at most only one generator (corresponding to the lines passing through that vertex) that may not commute with $x$ and now we can continue using the same methods of Step 4.7 till we cover all the vertices of the cycle of $G(\mathcal{A})$. Thus $\pi_{1}\left(\mathbb{C}^{2}-\left(\mathcal{A}^{\prime} \cup L_{3}\right)\right)$ has a conjugation-free presentation. In the same way, we can add the lines $L_{4}, \ldots, L_{k}$ inductively and get that $\pi_{1}\left(\mathbb{C}^{2}-\mathcal{A}\right)$ has a conjugation-free presentation.

The above proof implies that the conic contributes only one generator to the fundamental group, denoted by $x$ and it commutes with any other generator, corresponding to a line in the arrangement, i.e.:

Proposition 5.9 Let $\mathcal{A}$ be a real CL arrangement with one conic $C$. If $\mathcal{A}$ satisfies the conditions of Proposition 5.8, then: 


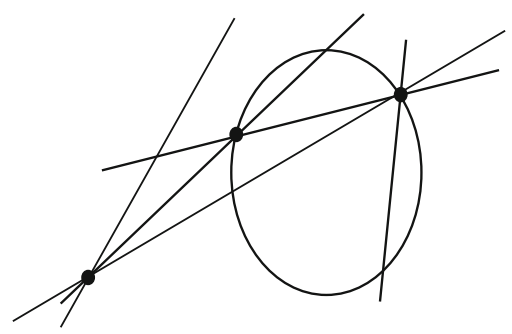

Fig. 23 An example of a CL arrangement with multiple points on the conic

$$
\pi_{1}\left(\mathbb{C}^{2}-\mathcal{A}\right) \cong\langle x\rangle \oplus \pi_{1}\left(\mathbb{C}^{2}-(\mathcal{A}-C)\right),
$$

where $x$ is the generator associated to the conic.

Remark 5.10 (1) Note that if $\mathcal{A}$ is a CL arrangement satisfying the conditions of Proposition 5.8 and $G(\mathcal{A}-C)$ has no cycles, then we know that $\pi_{1}\left(\mathbb{C}^{2}-(\mathcal{A}-C)\right)$ is a direct sum of free groups and a free abelian group (see $[13,17])$, and thus, by Proposition 5.9, $\pi_{1}\left(\mathbb{C}^{2}-\mathcal{A}\right)$ is also a direct sum of free groups and a free abelian group.

(2) The class of CL arrangements with a conjugation-free geometric presentation is larger than stated in Proposition 5.8. Indeed, it is easy to see that if the graph of a CL arrangement with one conic is a disjoint union of cycles, then the arrangement has a conjugation-free geometric presentation as well.

See also Sect. 6, where we define the notion of a conjugation-free graph, and show that if the associated graph of the arrangement is a conjugation-free graph, then the fundamental group of the arrangement has a conjugation-free geometric presentation.

Example 5.11 Based on Remark 5.10(1), the affine fundamental group of the complement of the CL arrangement in Fig. 23 is isomorphic to $\mathbb{Z}^{2} \oplus \mathbb{F}_{2} \oplus \mathbb{F}_{2}$.

\section{Conjugation-free graphs}

Following Remark 5.10(2) and the methods presented in the previous sections, this section examines a subclass of graphs, associated to a real line arrangement or to a real CL arrangement, which implies that the fundamental group of the arrangement has a conjugation-free geometric presentation.

6.1 A conjugation-free graph for real line arrangements

We start with the case of real line arrangements; Recall Proposition 2.18(1):

Proposition 6.1 Let $\mathcal{L}$ be a real line arrangement such that $\pi_{1}\left(\mathbb{C}^{2}-\mathcal{L}, u\right)$ has a conjugation-free geometric presentation for any real basepoint $u \in \ell-N$, where $\ell$ is the reference line. Let $L$ be a real line not in $\mathcal{L}$ that passes through at most one 


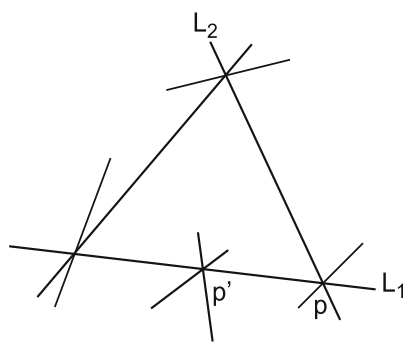

Fig. 24 For the point $p$, the lines $L_{1}$ and $L_{2}$ are drawn; for the point $p^{\prime}$, only the line $L_{1}$ is drawn

intersection point of $\mathcal{L}$. Then $\pi_{1}\left(\mathbb{C}^{2}-(\mathcal{L} \cup L), u\right)$ has a conjugation-free geometric presentation for any real basepoint $u$.

We can use this proposition to reprove the main result of [10, Proposition 1.4] in an inductive way:

Proposition 6.2 Let $\mathcal{L}$ be a real line arrangement. If $G(\mathcal{L})$ is a cycle, then $\pi_{1}\left(\mathbb{C}^{2}-\mathcal{L}\right)$ has a conjugation-free geometric presentation.

Proof We build $\mathcal{L}$ inductively: at each step, we prove that the fundamental group is conjugation-free.

(1) First, draw only the lines that correspond to the edges of the cycle $G(\mathcal{L})$ : denote by $\mathcal{L}_{0}$ the resulting arrangement. Since $G(\mathcal{L})$ is a cycle, then $G\left(\mathcal{L}_{0}\right)$ is the empty graph, as all the intersection points of $\mathcal{L}_{0}$ are nodes. Thus $\pi_{1}\left(\mathbb{C}^{2}-\mathcal{L}_{0}\right)$ is abelian and it is conjugation-free for any basepoint.

(2) Denote by $x_{1}, \ldots, x_{s}$ the nodes of $\mathcal{L}_{0}$ such that their associated vertex appeared in the graph $G(\mathcal{L})$ (i.e. the points correspond to the vertices of $G(\mathcal{L})$ ). Looking at the arrangement $\mathcal{L}$, there are $k_{i}$ lines passing through the point $x_{i}: L_{i_{1}}, \ldots, L_{i_{k_{i}}}$. Note that in $\mathcal{L}_{0}$, for each $x_{i}$ either one or two lines from the set $\left\{L_{i_{j}}\right\}_{j=1}^{k_{i}}$ were drawn (see Fig. 24).

Look at the point $x_{1}$ and assume that $L_{1_{1}}$ and $L_{1_{2}}$ (or $L_{1_{1}}$ ) were already drawn while building $\mathcal{L}_{0}$. Let $\mathcal{L}_{1} \doteq\left\{L_{1_{1}}, L_{1_{2}}\right\}$ (or $\mathcal{L}_{1} \doteq\left\{L_{1_{1}}\right\}$ resp.). Then $\mathcal{L}_{1}^{\prime} \doteq\left\{L_{1_{j}}\right\}_{j=1}^{k_{i}}-\mathcal{L}_{1}$ is a set of lines such that for each $L \in \mathcal{L}_{1}^{\prime}, L \cap\left\{x_{i}\right\}_{i=1}^{s}=x_{1}$; otherwise (explicitly, if $\left.L \cap\left\{x_{i}\right\}=\left\{x_{1}, x_{m}\right\}, m \notin\{2, s\}\right)$ we would get that $\beta(\mathcal{L})>1$. Note that if $m=2$ or $m=s$, then $L$ would be already in $\mathcal{L}_{1}$ (which is not possible, as $L \in \mathcal{L}_{1}^{\prime}$ ).

Therefore, each $L \in \mathcal{L}_{1}^{\prime}$ passes through a single intersection point of $\mathcal{L}_{0}$ and we can inductively add all the lines $L \in \mathcal{L}_{1}^{\prime}$ to $\mathcal{L}_{0}$ and preserve the conjugation-free property, by Proposition 6.1.

(3) We continue as in step (2) for the points $x_{2}, \ldots, x_{s}$, adding the missing lines. At each step, the conjugation-free property is preserved.

Recall the following result, stated in [11, Corollary 2.5]:

Proposition 6.3 Let $\mathcal{L}$ be a real line arrangement satisfying $\beta(\mathcal{L}) \leq 1$. Then, $\pi_{1}\left(\mathbb{C}^{2}-\mathcal{L}\right)$ has a conjugation-free geometric presentation. 


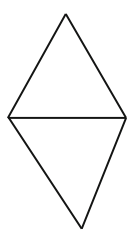

(a)

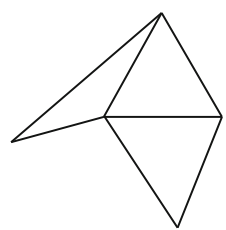

(b)

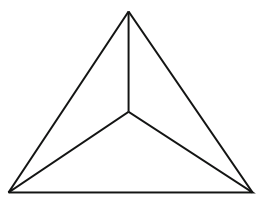

(c)

Fig. 25 Graphs a and $\mathbf{b}$ are conjugation-free graphs. Graph $\mathbf{c}$ is not a conjugation-free graph

Proof Indeed, this was already proved in [11], and we review the proof shortly. For the case $\beta(\mathcal{L})=0$, this is the content of Fan's result [14]: in this case, the fundamental group is a direct sum of a free abelian group and free groups and it is easy to see that it is conjugation-free for any basepoint. When $\beta(\mathcal{L})=1$, we can continue the construction above: all one needs to do is to add the lines corresponding to the trees whose roots lie on the unique cycle of $G(\mathcal{L})$.

Note that it is shown in [11] that if the graph is a disjoint union of cycles, then the arrangement is also conjugation-free, based on Oka-Sakamoto's theorem (see Theorem $5.3)$.

The above construction motivates the following definition:

Definition 6.4 Let $G$ be a planar connected graph and denote by $\operatorname{deg}(v)$ the number of edges exiting from a vertex $v \in G$. The graph $G$ is called a conjugation-free graph (CFG) if:

(1) $\beta(G) \leq 1$, or

(2) Let $\left\{v_{i}\right\}_{i=1}^{m}$ be the set of vertices in $G$ satisfying $\operatorname{deg}\left(v_{i}\right) \leq 2$. For each $v_{i}, 1 \leq i \leq m$, denote by $V_{i}$ the subset of $G$, composed of the vertex $v_{i}$ and the edge(s) exiting from it. Let $X=X(G) \doteq \bigcup_{i=1}^{m} V_{i}$. Then $G$ is a conjugationfree graph if $G-X$ is.

The main result of this section is:

Theorem 6.5 Let $\mathcal{L}$ be a real line arrangement. If $G(\mathcal{L})$ is a disjoint union of conjugation-free graphs, then $\pi_{1}\left(\mathbb{C}^{2}-\mathcal{L}\right)$ has a conjugation-free geometric presentation.

Before proving the theorem, we give some examples of conjugation-free graphs.

Example 6.6 (1) Obviously, a forest is a CFG.

(2) Graphs (a) and (b) in Fig. 25 are CFGs.

(3) The Ceva arrangement (also called the braid arrangement) has a non conjugationfree graph (see Fig. 25c).

Remark 6.7 Using the package TESTISOM (see [19]), one can show that there exist arrangements having a conjugation-free presentation for their fundamental group, though their associated graph is not a conjugation-free graph, e.g. the graph presented in Fig. 26. 


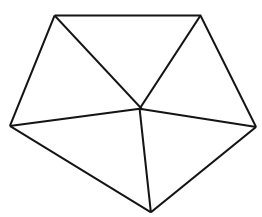

Fig. 26 A graph which is not a conjugation-free graph, though the fundamental group of its associated line arrangement has a conjugation-free geometric presentation

Proof of Theorem 6.5 We start with the case where $\mathcal{L}$ is a real line arrangement whose graph $G(\mathcal{L})$ is a connected conjugation-free graph. If $\beta(\mathcal{L}) \leq 1$, then the theorem holds due to Proposition 6.3. Assume now that $\beta(\mathcal{L})>1$. Denote $G=G(\mathcal{L})$ and $X=X(G)$ (the set of edges and vertices defined in Definition 6.14). We split our treatment into two cases.

Case (1): Assume that the graph $G-X$ has at most one cycle. Let $V=\left\{v_{1}, \ldots, v_{s}\right\} \subset X$ be the set of vertices (of $G$ ) which were removed. We now look at the sub-arrangement $\mathcal{L}_{X}$ of $\mathcal{L}$, which is the line arrangement associated to the graph $G-X$; i.e.

$$
\mathcal{L}_{X}=\mathcal{L}-\left\{\text { lines that pass through } v_{i}, 1 \leq i \leq s\right\}
$$

As $\beta\left(\mathcal{L}_{X}\right) \leq 1$, the fundamental group of the complement of $\mathcal{L}_{X}$ is conjugation-free for any basepoint. Now, for each $v_{i} \in V \subset X$, there are either one or two lines in $\mathcal{L}$, that pass through $v_{i}$, which correspond to the removed edges in the graph $G$. Now, add these lines to the arrangement $\mathcal{L}_{X}$ and call the new arrangement $\mathcal{L}_{X}^{\prime}$. Since these lines pass through at most one multiple point, then $\mathcal{L}_{X}^{\prime}$ is also conjugation-free, by Proposition 6.1.

Note that the every line in $\mathcal{L}-\mathcal{L}_{X}^{\prime}$ does not correspond to an edge in the graph. Therefore it passes through only one multiple point of $\mathcal{L}-\mathcal{L}_{X}^{\prime}$, and thus also $\mathcal{L}$ is conjugation-free.

Case (2): Assume now that the graph $G-X$ has more than one cycle. Let $G_{0}=G, X_{0}=X, G_{1}=G_{0}-X_{0}, X_{1}=X\left(G_{1}\right), \ldots, G_{n}=G_{n-1}-X_{n-1}$. Since $G_{0}$ is a CFG, then there is $k \in \mathbb{N}$ such that the graph $G_{k}$ has at most one cycle. In this case, we proceed inductively: in the first step, starting from the graph $G_{k}$, we add to the arrangement corresponding to the graph $G_{k}$ (which is conjugation-free) the lines that were removed from it in the last step (i.e. the lines that pass through all the vertices with degree 2 in the graph $G_{k-1}$ ). As was proved in case (1), we get an arrangement $\mathcal{L}_{k-1}$ whose fundamental group has a conjugation-free geometric presentation and its graph is $G_{k-1}$. Then we add to $\mathcal{L}_{k-1}$ its missing lines and we continue till we rebuild the whole arrangement $\mathcal{L}$.

The above two cases finalize the proof for the case of a real line arrangement $\mathcal{L}$, whose graph $G(\mathcal{L})$ is a connected conjugation-free graph. The general case of a real line arrangement $\mathcal{L}$, whose graph $G(\mathcal{L})$ is a disjoint union of conjugation-free graphs, can be deduced by Oka-Sakamoto's theorem (see [25] and Theorem 5.3 above). 
We finish this section by looking at another operation $h_{r}$ : rotation of the arrangement $\mathcal{L}$, when the reference line $\ell$ (and the real basepoint $u$ on it) remains fixed. We claim that when a real line arrangement $\mathcal{L}$ can be built by adding one line that passes through at most one intersection point at each step of its construction (i.e. the graph of $\mathcal{L}$ is a conjugation-free graph), then $h_{r}$ preserves the conjugation-free property.

Lemma 6.8 Let $\mathcal{L}$ be a real line arrangement such that $G(\mathcal{L})$ is a conjugation-free graph. Then, on this class of real line arrangements, the operation $h_{r}$ preserves the conjugation-free property for $\mathcal{L}$; that is, both fundamental groups $\pi_{1}\left(\mathbb{C}^{2}-\mathcal{L}, u\right)$ and $\pi_{1}\left(\mathbb{C}^{2}-h_{r}(\mathcal{L}), u\right)$ are conjugation-free.

Proof The proof is fairly trivial. We first draw the maximal number of lines of $\mathcal{L}$ such that its graph is empty, i.e. the resulting arrangement $\mathcal{L}_{0}$ consists only of nodes. Obviously, $\pi_{1}\left(\mathbb{C}^{2}-\mathcal{L}_{0}, u\right)$ is conjugation-free, as it is abelian, for every basepoint $u$. It is also clear that for the rotated arrangement $h_{r}\left(\mathcal{L}_{0}\right), \pi_{1}\left(\mathbb{C}^{2}-h_{r}\left(\mathcal{L}_{0}\right), u\right)$ is conjugation-free for every basepoint $u$. Now, for both arrangements, we can draw one line at each step of the construction, passing through at most one intersection point. By Lemma 3.6, at each step we still get that the two resulting arrangements have fundamental groups which are conjugation-free, and thus, eventually after drawing all the lines (in $\mathcal{L}-\mathcal{L}_{0}$ and in $h_{r}(\mathcal{L})-h_{r}\left(\mathcal{L}_{0}\right)$ ), we get that both fundamental groups have the conjugation-free property.

Remark 6.9 While other lemmata (such as Lemmas 3.3 and 3.4) prove the preservation of the conjugation-free property by finding the explicit isomorphism, the above lemma does not have to find it (even though the presentations of both fundamental groups are different), as we are using only the property that adding a line (that pass through at most one intersection point) preserves the conjugation-free property.

\subsection{A conjugation-free graph for real CL arrangements}

In this section, we check which results from Sect. 6.1 can be adapted to the case of real CL arrangements.

Note that if $\mathcal{A}$ is a real CL arrangement with only one conic, then the definition of a conjugation-free graph can be applied with some changes. Explicitly:

Definition 6.10 Let $G$ be a planar connected graph and let $\mathcal{A}$ be a real CL arrangement with one conic $C$. The graph $G$ is called a conjugation-free graph (CFG) associated to $\mathcal{A}$ if $G=G(\mathcal{A})$ and:

(1) $\beta(G)=0$, or $\beta(G)=1$ and $G$ satisfies Condition (2) of Proposition 5.8, or

(2) Let $\left\{v_{i}\right\}_{i=1}^{m}$ be the set of all vertices in $G$ satisfying $\operatorname{deg}\left(v_{i}\right) \leq 2$ and the point corresponds to the vertex $v_{i}$ is not on $C$. For each $v_{i}, 1 \leq i \leq m$, denote by $V_{i}$ the subset of $G$, composed of the vertex $v_{i}$ and the edge(s) exiting from it. Let $X=X(G) \doteq \bigcup_{i=1}^{m} V_{i}$. Then $G$ is a conjugation-free graph if $G-X$ is.

Indeed, assume that we are given a real $\mathrm{CL}$ arrangement $\mathcal{A}$ with only one conic $C$ and the graph $G(\mathcal{A})$ is a conjugation-free graph. Given an arrangement $\mathcal{A}^{\prime}$, such that 
$C \in \mathcal{A}^{\prime}$, with a graph $G^{\prime} \subset G$ with $\beta\left(G^{\prime}\right)=0$, or $\beta\left(G^{\prime}\right)=1$ and $G^{\prime}$ satisfies the conditions of Proposition 5.8(2), we can add the "missing" lines (i.e. all the lines in $\mathcal{A}-\mathcal{A}^{\prime}$ ) to this arrangement, in the same way described in the proof of Theorem 6.5, and the conjugation-free property of this arrangement is preserved (since all the lines intersect the conic in nodes, as we pass them through points not on $C$, we can use the same arguments that were used in the proof of Proposition 5.8).

Note that adding a conic $C_{2}$, passing through a single intersection point, to a $\mathrm{CL}$ arrangement $\mathcal{A}$ with one conic $C_{1}$, which has a conjugation-free graph associated to it, also preserves the conjugation-free property, under the following condition: Let $C_{1}, C_{2}$ be the two conics of the CL arrangement $\mathcal{A} \cup C_{2}$. For $i=1,2$, let $V_{i}$ be the set of vertices of $G\left(\mathcal{A} \cup C_{2}\right)$ which lie on the conic $C_{i}$. If $G\left(\mathcal{A} \cup C_{2}\right)$ is a disjoint union of two conjugation-free graphs $G_{1}, G_{2}$ such that $V_{i} \subset G_{i}$, then $\pi_{1}\left(\mathbb{C}^{2}-\left(\mathcal{A} \cup C_{2}\right)\right)$ has a conjugation-free geometric presentation (by Corollary 5.4).

We finish this section with the following conjecture, related to real line arrangements with a fundamental group having a conjugation-free geometric presentation:

Conjecture 6.11 Let $\mathcal{L}$ be a real line arrangement such that $\pi_{1}\left(\mathbb{C}^{2}-\mathcal{L}\right)$ has a conjugation-free geometric presentation, whose associated graph might not be a conjugation-free graph. Let $C$ be a conic that passes through a single intersection point of $\mathcal{L}$. Then $\pi_{1}\left(\mathbb{C}^{2}-(\mathcal{L} \cup C)\right)$ has a conjugation-free geometric presentation as well.

Acknowledgments We would like to thank Meital Eliyahu for stimulating talks. Also, we would like to thank an anonymous referee of a previous version of this paper for useful and important suggestions, especially regarding the proof of the preservation of the conjugation-free property. The first author would also like to thank especially Mikhail Zaidenberg from the Institut Fourier, Grenoble, for the numerous fruitful and productive talks. The first author would like to thank the Max-Planck-Institute für Mathematik in Bonn for the warm hospitality and support and the Fourier Institut in Grenoble, where the final part of this paper was carried out.

\section{References}

1. Amram, M., Garber, D., Teicher, M.: Fundamental groups of tangented conic-line arrangements with singularities up to order 6. Math. Zeit. 256, 837-870 (2007)

2. Amram, M., Teicher, M.: Fundamental groups of some special quadric arrangements. Rev. Mat. Comput. 19(2), 259-276 (2006)

3. Amram, M., Teicher, M., Uludag, A.M.: Fundamental groups of some quadric-line arrangements. Topol. Appl. 130(2), 159-173 (2003)

4. Artal-Bartolo, E.: Fundamental group of class of rational cuspidal curves. Manuscripta Math. 93, 273-281 (1997)

5. Artal-Bartolo, E.: A curve of degree five with non-abelian fundamental group. Topol. Appl. 83, 13-29 (1997)

6. Artal-Bartolo, E., Carmona-Ruber, J.: Zariski pairs, fundamental groups and Alexander polynomials. J. Math. Soc. Jpn. 50(3), 521-543 (1998)

7. Artal-Bartolo, E., Cogolludo, J.I., Tokunaga, H.: A survey on Zariski pairs. In: Algebraic Geometry in East Asia, Hanoi 2005, Adv. Stud. Pure Math., vol. 50, pp. 1-100. Math. Soc. Japan, Tokyo (2008).

8. Degtyarev, A.I.: Quintics in $\mathbb{C P}^{2}$ with nonabelian fundamental group, Algebra i Analiz 11(5), 130-151 (1999) [Russian]. English translation: St. Petersburg Math. J. 11(5), 809-826 (2000)

9. Dethloff, G., Orevkov, S., Zaidenberg, M.: Plane curves with a big fundamental group of the complement. In: Kuchment, P., Lin, V. (eds.) Voronezh Winter Mathematical Schools: Dedicated to Selim Krein, American Mathematical Society Translations-Series 2, vol. 184, pp. 63-84 (1998). 
10. Eliyahu, M., Garber, D., Teicher, M.: A conjugation-free geometric presentation of fundamental groups of arrangements. Manuscripta Math. 133(1-2), 247-271 (2010)

11. Eliyahu, M., Garber, D., Teicher, M.: A conjugation-free geometric presentation of fundamental groups of arrangements II: expansion and some properties. Int. J. Alg. Comput. 21(5), 775-792 (2011)

12. Eliyahu, M., Liberman, E., Schaps, M., Teicher, M.: Characterization of line arrangements for which the fundamental group of the complement is a direct product. Alg. Geom. Topol. 10, 1285-1304 (2010)

13. Fan, K.M.: Position of singularities and fundamental group of the complement of a union of lines. Proc. Am. Math. Soc. 124(11), 3299-3303 (1996)

14. Fan, K.M.: Direct product of free groups as the fundamental group of the complement of a union of lines. Mich. Math. J. 44(2), 283-291 (1997)

15. Friedman, M., Garber, D.: On the structure of fundamental groups of conic-line arrangements having a cycle in their graph. Topo. Appl. (2014). doi:10.1016/j.topol.2014.05.013

16. Friedman, M., Teicher, M.: The regeneration of a 5-point. Pure Appl. Math. Q. (Fedor Bogomolov special issue, part I), 4(2), 383-425 (2008).

17. Garber, D.: On the connection between affine and projective fundamental groups of line arrangements and curves. In: Brasselet, J.-P., Suwa, T. (eds.) Singularités Franco-Japonaises. Séminaires \& Congrès, vol. 10, pp. 61-70 (2005).

18. Garber, D., Teicher, M., Vishne, U.: Classes of wiring diagrams and their invariants. J Knot Theory Ramif 11(8), 1165-1191 (2002)

19. Holt, D.F., Rees, S.E.: The isomorphism problem for finitely presented groups. In: Groups, Combinatorics and Geometry, London Math. Soc. Lect. Notes Ser. 165, 459-475 (1992).

20. Jiang, T., Yau, S.S.-T.: Diffeomorphic types of the complements of arrangements of hyperplanes. Compositio Math. 92(2), 133-155 (1994)

21. Milnor, J.: Morse theory. Ann. Math. Stud. vol. 51. Princeton University Press, Princeton (1963).

22. Moishezon, B., Teicher, M.: Braid group technique in complex geometry, I, line arrangements in $\mathbb{C P}^{2}$. Contemp. Math. 78, 425-555 (1988)

23. Moishezon, B., Teicher, M.: Braid group technique in complex geometry, II, from arrangements of lines and conics to cuspidal curves. Algebraic Geom. Lect. Notes Math. 1479, 131-180 (1990)

24. Namba, M., Tsuchihashi, H.: On the fundamental groups of Galois covering spaces of the projective plane. Geom. Dedicata 104(1), 97-117 (2004)

25. Oka, M., Sakamoto, K.: Product theorem of the fundamental group of a reducible curve. J. Math. Soc. Jpn. 30(4), 599-602 (1978)

26. Orlik, P., Terao, H.: Arrangements of Hyperplanes, Grundlehren der Mathematischen Wissenschaften, vol. 300 (1992).

27. Tokunaga, H.: Sections of elliptic surfaces and Zariski pairs for conic-line arrangements via dihedral covers. J. Math. Soc. Japan 66(2), 613-640 (2014)

28. van Kampen, E.R.: On the fundamental group of an algebraic curve. Am. J. Math. 55, 255-260 (1933)

29. Wang, S., Yau, S.S.-T.: Rigidity of differentiable structure for new class of line arrangements. Comm. Anal. Geom. 13(5), 1057-1075 (2005)

30. Zariski, O.: On the problem of existence of algebraic functions of two variables possessing a given branch curve. Am. J. Math. 51, 305-328 (1929)

31. Zariski, O.: Algebraic Surfaces, 2nd edn. Springer, Heidelberg (1971) 